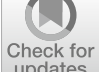

Check for
updates

Cite as

Nano-Micro Lett.

(2021) $13: 144$

Received: 17 April 2021

Accepted: 20 May 2021

Published online: 15 June 2021

(C) The Author(s) 2021

\section{Structural Engineering of Hierarchical Aerogels Comprised of Multi-dimensional Gradient Carbon Nanoarchitectures for Highly Efficient Microwave Absorption}

\author{
Yongpeng Zhao ${ }^{1,2}$, Xueqing Zuo ${ }^{1}$, Yuan Guo ${ }^{1}$, Hui Huang ${ }^{1}$, Hao Zhang ${ }^{1}$, Ting Wang ${ }^{1}$, \\ Ningxuan Wen ${ }^{1}$, Huan Chen ${ }^{1,2}$, Tianze Cong ${ }^{1}$, Javid Muhammad ${ }^{1}$, Xuan Yang ${ }^{3}$, \\ Xinnan Wang ${ }^{4}$, Zeng Fan $^{1} \bowtie$, Lujun $\operatorname{Pan}^{1} \bowtie$
}

\title{
HIGHLIGHTS
}

- The delicate "3D helix-2D sheet-1D fiber-0D dot" hierarchical aerogels were successfully synthesized.

- The graphene sheets are uniformly intercalated by helical carbon nanocoils, which endow the as-obtained aerogel with abundant porous structures and better dielectric properties.

- By adjusting the growth parameters of $0 \mathrm{D}$ core-shell structured particles and 1D carbon nanofibers, the tunable electromagnetic properties and excellent impedance matching are achieved.

ABSTRACT Recently, multilevel structural carbon aerogels are deemed as attractive candidates for microwave absorbing materials. Nevertheless, excessive stack and agglomeration for low-dimension carbon nanomaterials inducing impedance mismatch are significant challenges. Herein, the delicate "3D helix-2D sheet-1D fiber-0D dot" hierarchical aerogels have been successfully synthesized, for the first time, by sequential processes of hydrothermal self-assembly and in-situ chemical vapor deposition method. Particularly, the graphene sheets are uniformly intercalated by 3D helical carbon nanocoils, which give a feasible solution to the mentioned problem and endows the as-obtained aerogel with abundant porous structures and better dielectric properties. Moreover, by adjusting the content of OD core-shell structured particles and the parameters for growth of the 1D carbon

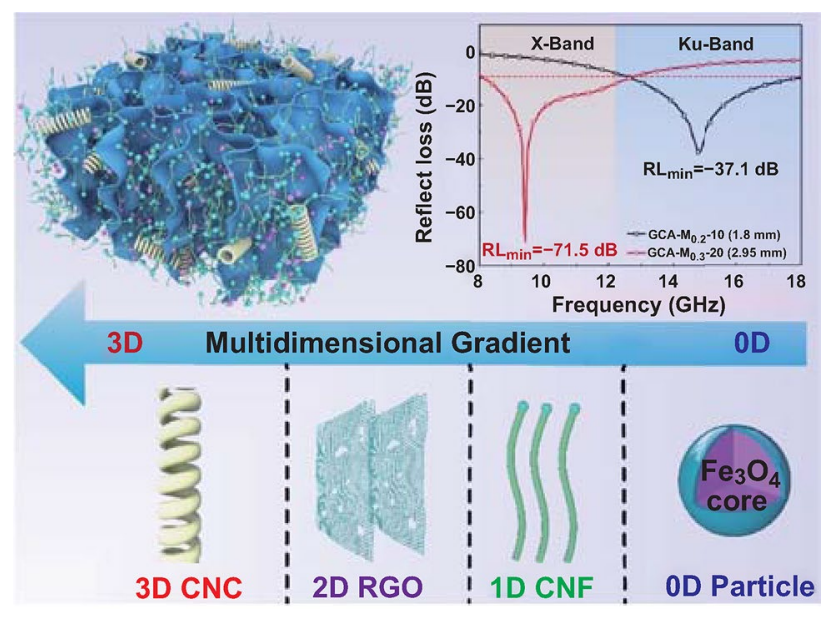
nanofibers, tunable electromagnetic properties and excellent impedance matching are achieved, which plays a vital role in the microwave absorption performance. As expected, the optimized aerogels harvest excellent performance, including broad effective bandwidth and strong reflection loss at low filling ratio and thin thickness. This work gives valuable guidance and inspiration for the design of hierarchical materials comprised of dimensional gradient structures, which holds great application potential for electromagnetic wave attenuation.

KEYWORDS Hierarchical aerogels; Multi-dimensional gradient; Carbon nanocoils; Microwave absorption

Zeng Fan, fanzeng@dlut.edu.cn; Lujun Pan, lpan@dlut.edu.cn

School of Physics, Dalian University of Technology, Dalian 116024, Liaoning, People's Republic of China

2 School of Microelectronics, Dalian University of Technology, Dalian 116024, Liaoning, People's Republic of China

3 School of Materials Science and Engineering, Dalian University of Technology, Dalian 116024, Liaoning, People's Republic of China

${ }^{4}$ School of Chemical Engineering, Dalian University of Technology, Dalian 116024, Liaoning, People's Republic of China 


\section{Introduction}

Nowadays, microwave absorbing materials (MWAMs) have received great interest for their wide applications in wireless communications, information processing and Radar stealth technologies [1-4]. To date, a large variety of traditional materials have been developed to be used as MWAMs, such as transition metals (Fe [5], Co [6], Ni [7], Mn [8], and their alloys, ferrites, and ceramics $[9,10])$. However, due to the large bulk densities, poor mechanical and chemical properties, the traditional materials hardly meet practical application requirements in future high-tech era. Thus, there has an urgent pursuit of advanced MWAMs with strong attenuation capability, wide effective bandwidth, low density, and thin thickness.

In general, microwave absorption performances of MWAMs are significantly linked with the parameters of complex permittivity and permeability of these materials. Therefore, an ingenious design for the special structures with tailorable electromagnetic parameters is a feasible strategy to upgrade their microwave absorption performance. Up to now, the porous [11-13], core-shell [14-18], yolk-shell $[19,20]$, and hierarchical-structured [21-28] materials have been certified as efficient MWAMs due to their special structures endowed with multiple microwave attenuation mechanisms. Among them, hierarchically structured materials are undoubtedly a kind of up-and-coming candidate for MWAMs due to their features of low density, multidimensional components, highly tunable dielectric loss, high specific surface area, and favorable impedance matching. For example, Che and his colleagues fabricated a 3D hierarchically structured $\mathrm{MoS}_{2} / \mathrm{FeS}_{2}$ composite, which showed an extended effective absorption bandwidth (EAB) of $6.48 \mathrm{GHz}$ with a filler loading ratio of $50 \mathrm{wt} \%$ [29]. Liu et al. synthesized a composite of carbon fiber@MXene@ $\mathrm{MoS}_{2}$, the minimum reflection loss $\left(\mathrm{RL}_{\text {min }}\right)$ value reached $-61.5 \mathrm{~dB}$ at a thickness of $3.5 \mathrm{~mm}$ [30]. Wu and his co-authors prepared an efficient EMAM of hierarchical cobalt nanocrystals/Ndoped carbon nanotubes (CNTs)/carbon sponge, the $\mathrm{RL}_{\text {min }}$ value reached $-52 \mathrm{~dB}$, and the $\mathrm{EAB}$ was $4.1 \mathrm{GHz}$ at the thickness of $2.2 \mathrm{~mm}$ [31]. Inspired by the excellent microwave absorption performances of these unique structures mentioned above, the novel architecture with hierarchical structure and tunable electromagnetic parameter is a promising way to upgrade the performance of EMAMs.
For decades, carbon-based microwave absorbing materials, such as 0D fullerene, 1D CNTs and nanofibers (CNFs), 2D graphene, and 3D carbon nanocoils (CNCs), have attracted tremendous attention due to their outstanding dielectric loss capacity, good corrosion resistance, high physical/chemical stability, and lightweight [32-34]. Meanwhile, it is worth noting that the differences in hybridization form of carbon atoms and morphology would affect the configurations and microwave absorbing properties of nanostructures. Typically, OD structured materials (fullerene or carbon-based particles) have the advantage of integrating the magnetic and dielectric materials [35]. 1D structured materials (CNTs or CNFs) have the advantages of high anisotropy and aspect ratio, which facilitate the conducting loss during carrier transporting [36]. Besides, 2D graphene consumes energy through polarization loss and multiple reflection loss caused by large specific surface area, abundant oxygen-containing functional groups and multilayer structure [37]. Notably, the latest researches have reported that hierarchical structures comprising multi-dimensional carbon materials, such as the hybrid of particles, CNTs, and graphene, exhibit enhanced microwave absorption performances. However, the excessive stack of graphene and the agglomeration of CNTs/CNFs would reduce the porosity and interfacial compatibility of composite, which cause the interfacial impedance mismatching [2]. On the other hand, higher conductivities of graphitized carbon nanomaterials (CNT, $\mathrm{CNF}$, and graphene) lead to skin effect that inhibits the further incidence of microwave into the EMAM. Therefore, it is very important to develop a promising method to tailor the conductivities of carbon-based hierarchical structures. To settle the tough challenges mentioned above, a novel structure and modified preparation strategy should be used. Interestingly, the CNCs give the new inspiration for designing novel composite materials due to its 3D morphology, chiral, and amorphous structure [38-42]. To date, the CNC-based composites have exhibited promising microwave attenuation capability. For example, Qin et al. prepared $\mathrm{Fe}_{3} \mathrm{O}_{4} / \mathrm{Al}_{2} \mathrm{O}_{3} / \mathrm{CNCs}$ by an atom layer deposition method, and the value of $\mathrm{RL}_{\text {min }}$ reached $-50.7 \mathrm{~dB}$ at the thickness of $2.4 \mathrm{~mm}$ [33]. Cui and co-authors prepared a CNCs/tissue composite, the $\mathrm{RL}_{\text {min }}$ value reached $-46.3 \mathrm{~dB}$, and the $\mathrm{EAB}$ was $7.4 \mathrm{GHz}$ at the thickness of $2 \mathrm{~mm}$ [43]. Recently, our group reported a 
core-shell structured $\mathrm{Fe}_{3} \mathrm{O}_{4} @ \mathrm{C} / \mathrm{CNC}$ absorber [44], and the $R L_{\min }$ reached $-47.5 \mathrm{~dB}$ at the thickness of $1.7 \mathrm{~mm}$, while the value of EAB reached $5.03 \mathrm{GHz}$ at the filler loading ratio of $40 \mathrm{wt} \%$. On the other hand, the unique $3 \mathrm{D}$ helical skeleton would alleviate the stacking of graphene layers and boost the porosity of the composite. More importantly, the chiral CNCs would induce cross-polarization loss, which makes the material possess outstanding dielectric loss performance [45]. Therefore, considering the issue of excessive stack and agglomeration for lowdimension carbon materials, as well as the challenge of impedance mismatch caused by excessive conductivity, it is a highly promising way to introduce the $3 \mathrm{D}$ amorphous CNCs into the hierarchical structure for further improvements of energy attenuation and impedance matching.

Herein, we report a scalable strategy to fabricate hierarchical aerogels composed of CNCs/graphene/CNFs/ coreshell particles with the "3D helix-2D sheet-1D fiber-0D dot" structure by using self-assembly and in-situ chemical vapor deposition (CVD) methods. The advantages of this work are summarized as follow. First, the 2D graphene layer is evenly inserted by the $3 \mathrm{D}$ spiral CNCs, which gives the aerogel the porous structure and better dielectric properties. Then, two kinds of metal oxide nanoparticles (M-NPs) play different roles in the following CVD process. The NiO nanoparticle is used as a catalyst to synthesize 1D structured CNFs. As a result, the confined CNFs in aerogel tailor the conductive loss, which would significantly upgrade the dielectric loss of material. Besides, the 0 $\mathrm{D} \mathrm{Fe}_{3} \mathrm{O}_{4} @ \mathrm{C}$ coreshell composites derived from $\mathrm{Fe}_{2} \mathrm{O}_{3}$ would not only endow the hybrid materials with the magnetic loss but also improve the impedance matching. Consequently, the as-prepared hierarchical structures deliver a strong attenuation capability and wide effective bandwidth at low filling ratio and thin thickness. This work would shed light on the delicate design of hierarchically structured aerogel for high-performance microwave absorption and other potential applications.

\section{Experimental Section}

The synthesis procedure of the hierarchical aerogel is schematically shown in Fig. 1. First, the reduced graphene oxide (RGO)/CNC/M-NPs aerogel was synthesized by a sequential process of hydrothermal and freeze-dry methods. Whereafter, the hierarchical RGO/CNC/CNF/M-NPs aerogels were obtained through the CVD process. By adjusting the CVD reaction time and the content of M-NPs, the samples with different electromagnetic properties were synthesized. A series of CNC-contained samples based on different synthesis conditions are labeled as GCA- $\mathrm{M}_{X}-Y(X=0.2,0.3 \mathrm{mmol}$, and $Y=0,5,10$, and $20 \mathrm{~min}$, respectively). The subscript $X$ in labels represents the total molar mass of M-NPs in the nanocomposite, while the $Y$ represents reaction time of CVD process. The additional control samples were also fabricated without the addition of CNCs. Under this condition, the samples are designated as $\mathrm{GA}-\mathrm{M}_{X}-Y$. For example, the sample GCA- $\mathrm{M}_{0.2}-10$ indicates the content of M-NPs in aerogel was $0.2 \mathrm{mmol}$, while the reaction time of the subsequent CVD process was $10 \mathrm{~min}$. The details of each synthesis procedure were as follows.

\subsection{Materials}

Graphene oxide powder was purchased from Nanjing XFNANO Materials. Besides, the CVD technology was used to synthesize high-purity CNCs by using porous $\mathrm{Fe}_{2} \mathrm{O}_{3} / \mathrm{SnO}_{2}$ nanoparticles as the catalyst. Specifically, the growth process was carried out at $710^{\circ} \mathrm{C}$ for 180 min with the introduction of 350 and $30 \mathrm{sccm} \mathrm{Ar}$ and $\mathrm{C}_{2} \mathrm{H}_{2}$ gases simultaneously [46, 47]. After reaction, the CNCs were dispersed and washed by $68 \mathrm{wt} \%$ nitric acid and deionized (DI) water successively. The $\mathrm{Fe}_{2} \mathrm{O}_{3}$ and $\mathrm{NiO}$ nanoparticles were synthesized by using one-step hydrothermal method with the same reducing agent and reaction condition. Typically, $0.2 \mathrm{mmol}$ soluble $\mathrm{Fe}^{3+}$ or $\mathrm{Ni}^{2+}$ salts were dissolved in $35 \mathrm{~mL}$ DI water, then $1.0 \mathrm{~g}$ urea was added into the mixture solution. After stirring vigorously for $30 \mathrm{~min}$, the mixture was transferred into a $50 \mathrm{~mL}$ Teflon-lined autoclave and maintained at $160{ }^{\circ} \mathrm{C}$ for $6 \mathrm{~h}$. The resulting precipitates were washed with DI water three times and then dried at $60{ }^{\circ} \mathrm{C}$ for $24 \mathrm{~h}$. Figure S1 shows the SEM images of all as-obtained precursors.

\subsection{Preparation of RGO/CNC/M-NPs Aerogels}

For the synthesis of RGO/CNC/M-NPs aerogels with different properties, A total of $X(X=0.2,0.3) \mathrm{mmol}$ of $\mathrm{Fe}_{2} \mathrm{O}_{3}$ and $\mathrm{NiO}$ mixed powders were added in $15 \mathrm{~mL}$ DI water, and the molar mass ratio of $\mathrm{Fe}_{2} \mathrm{O}_{3}$ and $\mathrm{NiO}$ was kept as 1:1. Afterward, $40 \mathrm{mg}$ graphene oxide and $10 \mathrm{mg} \mathrm{CNC}$ powders were added to 


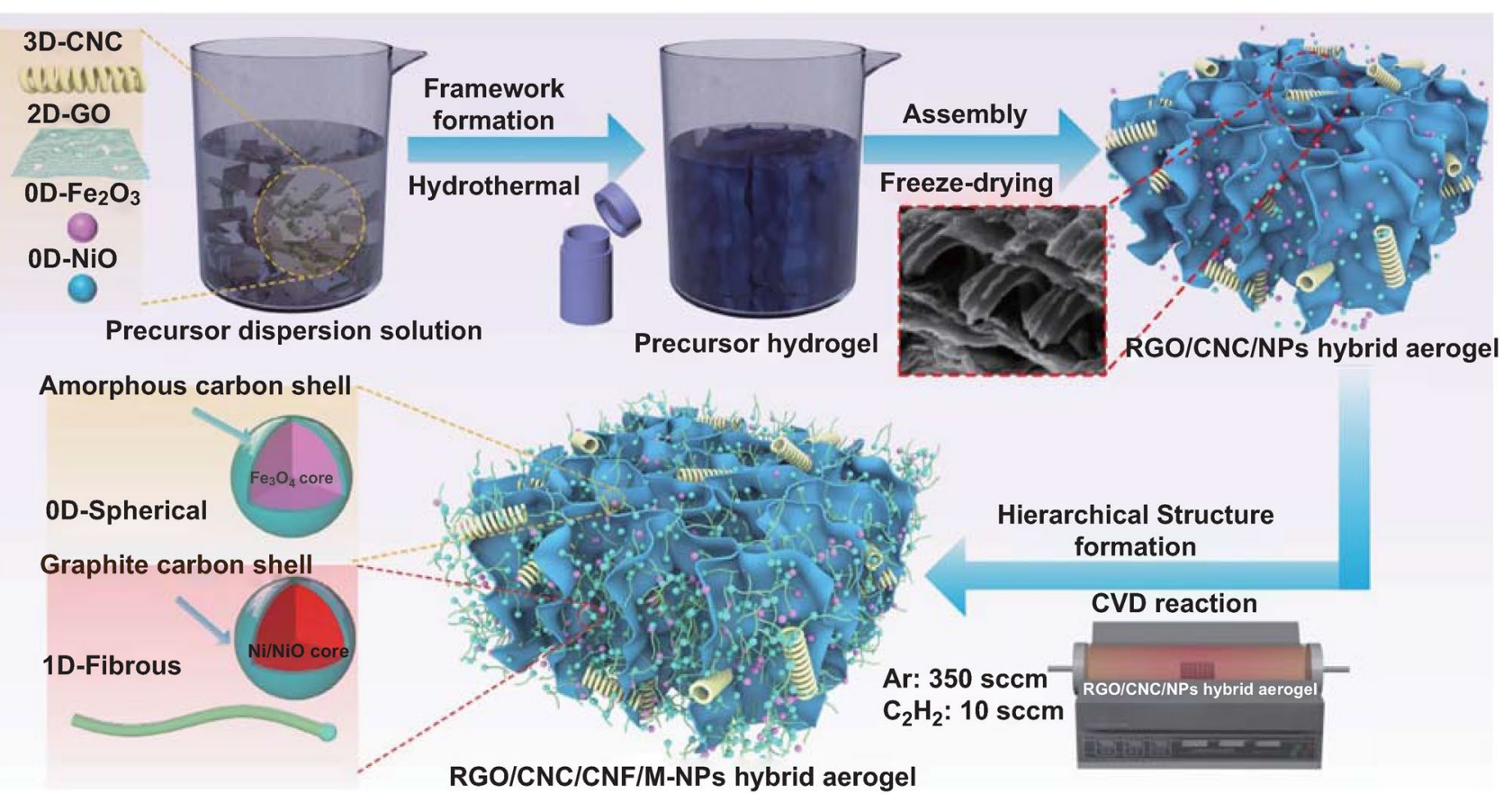

Fig. 1 Overall synthesis procedure of the RGO/CNC/CNF/M-NPs hierarchical aerogel

the mixture solution. After stirring and ultrasonication for $3 \mathrm{~h}$, the solution was transferred into a $25 \mathrm{~mL}$ Teflon-lined autoclave and maintained at $180{ }^{\circ} \mathrm{C}$ for $12 \mathrm{~h} \mathrm{[48].} \mathrm{Subsequently,}$ the resulting hydrogels were washed with deionized water and then freeze-dried at $-70{ }^{\circ} \mathrm{C}$ for $24 \mathrm{~h}$. When the contents of added M-NPs were 0.2 and $0.3 \mathrm{mmol}$, the as-obtained samples were designated as GCA- $\mathrm{M}_{0.2}-0$ and $\mathrm{GCA}-\mathrm{M}_{0.3}-0$, respectively. Meanwhile, additional control samples were also fabricated using $50 \mathrm{mg}$ graphene oxide powder (without CNCs) in the same preparation condition, and the as-prepared samples were labeled as $\mathrm{GA}-\mathrm{M}_{0.2}-0$ and $\mathrm{GA}-\mathrm{M}_{0.3}-0$, respectively. Figure $\mathrm{S} 2$ shows the SEM images of all as-obtained aerogels.

\subsection{Preparation of RGO/CNC/CNF/M-NPs Aerogels}

The RGO/CNC/CNF/M-NPs aerogels were synthesized by the CVD methods as follows. Prior to the process, $30 \mathrm{mg}$ RGO/CNC/M-NPs aerogel was uniformly supported by the quartz boat. To prepare aerogels with different electromagnetic properties, the reaction time was controlled precisely. Specifically, the process was carried out at $450{ }^{\circ} \mathrm{C}$ with the introduction of 350 and $10 \mathrm{sccm} \mathrm{Ar}$ and $\mathrm{C}_{2} \mathrm{H}_{2}$ gases simultaneously. When the reaction time was 5,10 , and $20 \mathrm{~min}$, the as-obtained samples were designated as GCA- $\mathrm{M}_{0.2}-5$, GCA- $\mathrm{M}_{0.2}-10$, GCA- $\mathrm{M}_{0.2}-20$, GCA- $\mathrm{M}_{0.3}-5$, GCA $-\mathrm{M}_{0.3}-10$, and GCA $-\mathrm{M}_{0.3}-20$, respectively.

\subsection{Characterization}

The morphologies of samples were investigated by fieldemission scanning electron microscope (FE-SEM, NOVA NanoSEM 450) operating at $3 \mathrm{kV}$, and transmission electron microscope (TEM) on a FEI Tecnai F30 operating at $300 \mathrm{kV}$. Meanwhile, the elemental mappings of the samples were also measured by energy dispersive X-ray spectrometers (EDX), which are equipped with the FE-SEM and TEM. The chemical composition of sample was identified by X-ray diffraction (XRD, PANalytical B.V diffractometer), X-ray photoelectron spectroscopy (XPS, VG ESCALAB 250Xi), and Raman spectroscopy (Renishaw in Via plus). The magnetic properties and surface areas of the samples were carried out by using a vibrating sample magnetometer (VSM, LakeShore-7300S) and a specific surface analyzer (QUADRASORB SI-KR/MP, Quantachrome, USA), respectively. The electrical conductivities of samples were measured using a classical 4-probe inline contact method with a Keithley 2450 source/meter. 


\subsection{Electromagnetic Measurements}

To measure the complex electromagnetic parameters, asobtained aerogels were crushed and mixed homogeneously with wax under the weight ratio of 15:85. Afterward, the mixture was pressed into a standard toroidal-shaped sample, with an inner diameter of $3.04 \mathrm{~mm}$, outer diameter of $7 \mathrm{~mm}$, and the thickness of $2 \mathrm{~mm}$. Afterward, the network analyzer (Agilent 8720B) was used to measure the electromagnetic parameters in the range of $1-18 \mathrm{GHz}$ at $293 \mathrm{~K}$.

To evaluate the performance of the as-obtained EMAMs, the RL values in the frequency of $1-18 \mathrm{GHz}$ were calculated on the basis of transmission line theory, which was depicted by Eqs. (1) and (2) [49-62]:

$\mathrm{RL}=20 \log \left|\left(Z_{\text {in }}-Z_{0}\right) /\left(Z_{\text {in }}+Z_{0}\right)\right|$

$Z_{\text {in }}=Z_{0} \sqrt{\frac{\mu_{\mathrm{r}}}{\varepsilon_{\mathrm{r}}}} \tanh \left[\frac{j(2 \pi f d)}{c \sqrt{\varepsilon_{\mathrm{r}} \mu_{\mathrm{r}}}}\right]$

where $Z_{0}$ and $Z_{\text {in }}$ are the impedance of free space and the normalized input impedance of absorber, respectively. Besides, the $f$ and $c$ represent the frequency of the microwave and the speed of light, respectively. Finally, $d$ is the thickness of the absorber.

\section{Result and Discussion}

\subsection{Morphology and Composition}

The morphologies and typical properties of as-obtained aerogel were investigated. Figure 2a shows an optical image of a GCA- $\mathrm{M}_{0.2}-10$ aerogel supporting by a dandelion without any deformation of the stem, exhibiting the lightweight nature of the aerogel. Figures S3a and $2 b$ show the optical image of a GCA- $\mathrm{M}_{0.2}-0$ aerogel before and after the CVD reaction, respectively. These results indicate that the magnetic aerogel was prepared after the CVD process. Furthermore, Fig. $2 \mathrm{c}$, d display the morphologies of GCA- $\mathrm{M}_{0.2}-0$ and GCA- $\mathrm{M}_{0.3}-0$ aerogels, respectively. It is observed that the CNCs insert into the graphene layers, which boost the porosity of the samples. As a comparison, the RGO nanosheets in GA series samples (without CNCs) seriously stacked, and there are few porous structures in the samples (as indicated in Figs. S2a, b, e, and f). Meanwhile, by increasing the added content of M-NPs in the precursor, the nanoparticle density of GCA- $\mathrm{M}_{0.3}-0$ is obviously larger than that of GCA- $\mathrm{M}_{0.2}-0$. Figure $2 \mathrm{e}, \mathrm{f}$ shows the SEM images of GCA $-\mathrm{M}_{0.2}-10$, it is found that abundant short 1D CNFs and OD carbon particles emerge from the RGO/CNC skeleton. Figure $2 \mathrm{f}$ suggests the hierarchical aerogel comprising of 0-, 1-, 2-, and 3-D nano carbon cells maintains its porous structure well. With increasing the content of M-NPs and CVD reaction time, the aerogels with different morphologies were synthesized, as shown in Fig. 2g, h. It is observed that dense and long CNFs appear and almost fully cover the RGO/CNC skeleton. From the insert of Fig. 2h, it is seen that the typical $1 \mathrm{D} \mathrm{CNF}$ with a fiber diameter of about $45 \mathrm{~nm}$. A series of SEM analyses of the materials prepared under different conditions were conducted and given by Fig. S4. These results suggest that the growth density and length of CNFs increase with the increasing of the M-NPs content and CVD reaction time, respectively. The morphologies of the GA series aerogels after CVD reaction are observed by Fig. S5a, b. It is concluded that GCA series samples exhibit obvious porous structure than GA series samples. This result further proves that the introduction of $\mathrm{CNC}$ into the aerogel can increase porosity and give the necessary space for CNF growth. To explore the distributions of elements in GCA- $\mathrm{M}_{0.3}-20$, elemental distributions of carbon (C), iron (Fe), Nickel (Ni), and oxygen $(\mathrm{O})$ were further investigated using EDX mapping. As shown in Fig. 2i, the $\mathrm{Fe}$ and Ni elements are well distributed throughout the carbon network. The nitrogen adsorption/desorption measurements were utilized to analyze the specific surface areas and pore structure of typical samples. As shown in Fig. $2 \mathrm{j}$, the curves of GCA- $\mathrm{M}_{0.2}-10$ and GCA- $\mathrm{M}_{0.3}-20$ show an IV-type isotherm [63], and the long and narrow hysteresis loops at relative pressure from 0.45 to 1.0 are observed simultaneously. The calculated specific surface areas of GCA- $\mathrm{M}_{0.2}-10$ and GCA- $\mathrm{M}_{0.3}-20$ are 369.5 and $372.4 \mathrm{~cm}^{2} \mathrm{~g}^{-1}$, respectively. Besides, the pore distribution properties of the as-mentioned samples are given by Fig. 2k. It is confirmed that a nano- and mesoporous hybrid matrix exists in the samples [64]. Furthermore, as shown in Fig. S5e, the calculated specific surface areas of $\mathrm{GA}-\mathrm{M}_{0.2}-10$ and $\mathrm{GA}-\mathrm{M}_{0.3}-20$ are only 245.4 and $257.1 \mathrm{~cm}^{2} \mathrm{~g}^{-1}$, respectively. The above results well prove that the introduction of $\mathrm{CNC}$ would increase the porosity of aerogel. Meanwhile, a comparison table of specific surface area for RGO-based hierarchical materials is given in 

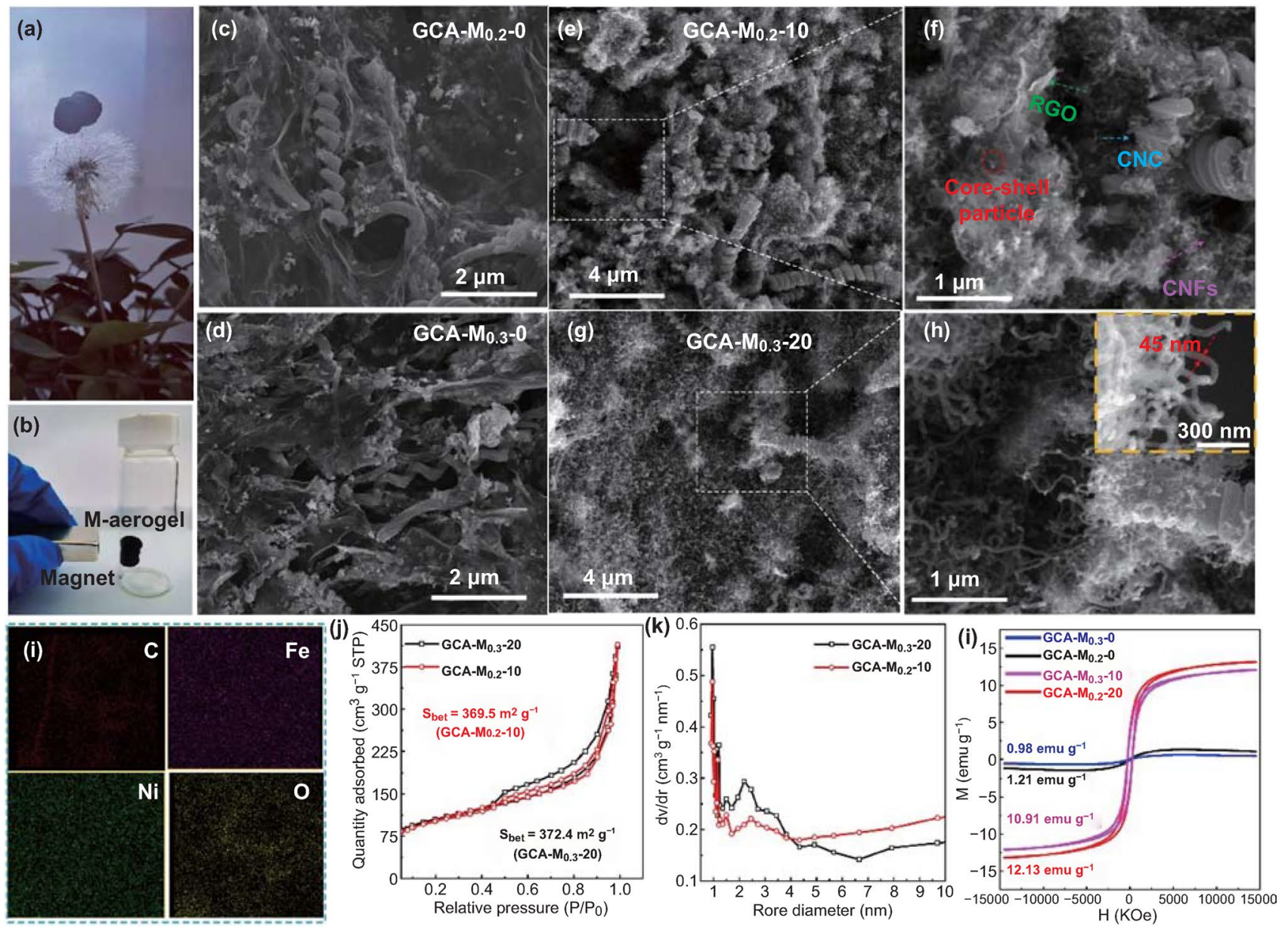

Fig. 2 As-obtained aerogel (a) standing on a dandelion, and (b) attracting by a magnet; The SEM images of (c) GCA-M $0.2-0$, (d) GCA-M $0.3-0$, $(\mathbf{e}, \mathbf{f}) \mathrm{GCA}-\mathrm{M}_{0.2}-10$, and $(\mathbf{g}, \mathbf{h}) \mathrm{GCA}-\mathrm{M}_{0.3}-20$, (i) elemental mappings of $\mathrm{C}, \mathrm{Fe}, \mathrm{Ni}$, and $\mathrm{O}$ in $\mathrm{GCA}-\mathrm{M}_{0.3}-20$. (j) $\mathrm{N}_{2}$ adsorption/desorption isotherms and (k) corresponding pore size distributions for the GCA-M $\mathrm{M}_{0.2}-10$ and GCA-M $\mathrm{M}_{0.3}-20$. (l) Magnetic hysteresis loops of GCA-M $\mathrm{M}_{0.2}-0$, GCA $-\mathrm{M}_{0.3}-0$, GCA $-\mathrm{M}_{0.2}-10$, and GCA- $\mathrm{M}_{0.3}-20$

Table S1. According to the comparison table, it is concluded that the use of CNC to decrease graphene stacking and agglomeration is an effective strategy. Furthermore, magnetic hysteresis loops of typical samples are given by Fig. 21, and the saturation magnetization values for $\mathrm{GCA}-\mathrm{M}_{0.2}-0$, GCA- $\mathrm{M}_{0.3}-0$, GCA $-\mathrm{M}_{0.2}-10$, and GCA- $\mathrm{M}_{0.3}-20$ are 0.98 , $1.21,10.91$, and $12.13 \mathrm{emu} \mathrm{g}^{-1}$, respectively. These results further certify that the non-magnetic sample is converted to a magnetic one after the CVD process, which endows asobtained aerogel with the magnetic loss.

The morphologies and internal structures of typical samples were systematically observed by TEM and HRTEM images. It is clearly seen that the GCA- $\mathrm{M}_{0.2}-0$ aerogel consists of graphene nanosheets, helical CNCs, and M-NPs (as shown in Fig. S6a). Meanwhile, it is certified that no CNFs or carbon shell (Fig. S6b) are synthesized before the CVD process. As displayed in Fig. 3a, the GCA- $\mathrm{M}_{0.2}-10$ aerogel with hierarchical and porous structure appeared, and some CNFs and nanoparticles are synthesized and deposited on the skeleton of RGO/CNC. The HRTEM images further confirm the internal structures of OD core-shell nanoparticles and 1D CNFs. In Fig. 3b, the HRTEM and its corresponding FFT images confirm that the interplanar spacing of $0.29 \mathrm{~nm}$ can correspond to the (220) plane of $\mathrm{Fe}_{3} \mathrm{O}_{4}$ [65]. Meanwhile, the thin layer of amorphous carbon covers the $\mathrm{Fe}_{3} \mathrm{O}_{4}$ core, which convincingly confirms the formation of core-shell structured $\mathrm{Fe}_{3} \mathrm{O}_{4} @$ C. Furthermore, as shown in Fig. 3c, the catalyst $\mathrm{Ni}$ particles are encapsulated on the top of short and 

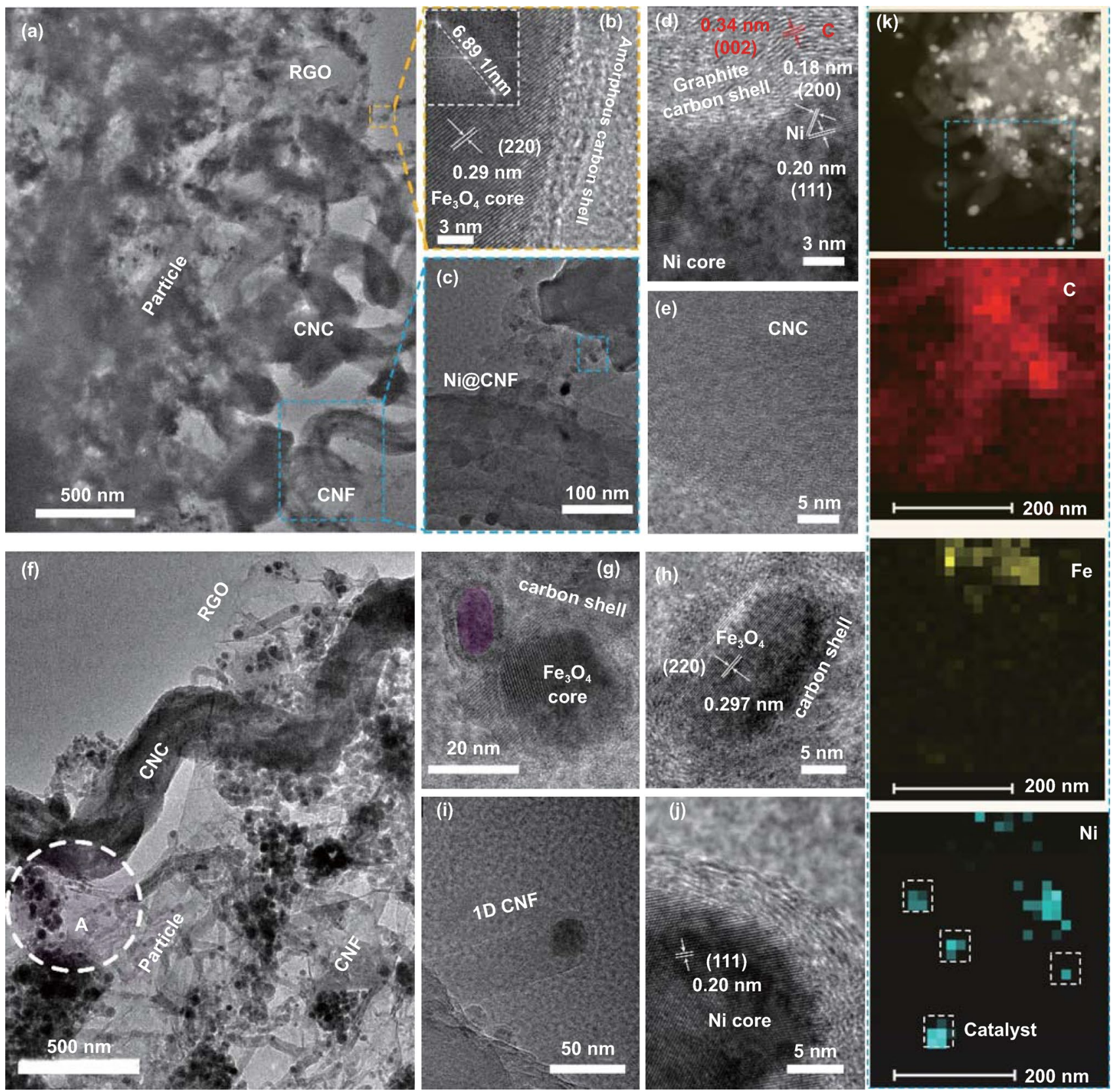

Fig. 3 (a) TEM image of GCA-M $\mathrm{M}_{0.2}-10$. Corresponding HRTEM images of (b) $\mathrm{Fe}_{3} \mathrm{O}_{4} @ \mathrm{C}$ (insert of b FFT image), (c, d) carbon nanofiber, and (e) CNC. (f) TEM image of GCA-M ${ }_{0.3}-20$. Corresponding HRTEM images of (g, h) $\mathrm{Fe}_{3} \mathrm{O}_{4} @ \mathrm{C}$ and (i, j) carbon nanofiber. (k) Elemental maps of $\mathrm{C}, \mathrm{Fe}$, and $\mathrm{Ni}$ in the $\mathrm{GCA}-\mathrm{M}_{0.3}-20$ sample

irregular CNFs. Besides, three crystal interplanar spacings (Fig. 3d), 0.18, 0.20, and $0.34 \mathrm{~nm}$, could be well fitted with the (200), (111) of Ni, and (002) of graphitic carbon, respectively [66].These results suggest that the $\mathrm{Fe}_{2} \mathrm{O}_{3}$ and $\mathrm{NiO}$ are partially reduced to $\mathrm{Fe}_{3} \mathrm{O}_{4}$ and $\mathrm{Ni}$ during the CVD process, which play the role in catalytically synthesizing the carbon shell and CNFs, respectively. Furthermore, the HRTEM image of $\mathrm{CNC}$ was given by Fig. 3e, it is observed that the crystallinity of the $\mathrm{CNC}$ is poor because the orientation of crystallites is disordered. For comparison, the TEM and HRTEM images of GCA-M $\mathrm{M}_{0.3}-20$ were given by Fig. $3 \mathrm{f}-\mathrm{j}$. As shown in Fig. 3f, the 3D CNC, 2D RGO, 1D CNFs, and 
OD M-NPs are obviously observed. With the increase in the reaction time, the $\mathrm{Fe}_{3} \mathrm{O}_{4} @ \mathrm{C}$ nanoparticle with a thicker carbon shell is seen in Fig. 3g, h. The area marked A in Fig. $3 \mathrm{f}$ is carefully observed. A large number of particles with a core-shell structure were confirmed in Fig. S7. Besides, the internal particle with a lattice spacing of $0.297 \mathrm{~nm}$ is well in line with the (220) plane of $\mathrm{Fe}_{3} \mathrm{O}_{4}$ (Fig. 3h). The HRTEM image of the catalyst particle is given by Fig. 3j, the typical lattice interlayer with the distance of $0.20 \mathrm{~nm}$ correspond to the (111) crystal plane of Ni [67]. Besides, to further elucidate the effects of $\mathrm{Fe}_{2} \mathrm{O}_{3}$ and $\mathrm{NiO}$ in the precursor, the EDX mapping images are used to investigate the element distributions of GCA-M $\mathrm{M}_{0.3}-20$. As shown in Fig. 3k, the distributions of $\mathrm{Ni}$ and $\mathrm{Fe}$ are obviously different. More importantly, when we check the tip area of the CNFs, only the signal of $\mathrm{Ni}$ appears, which strongly suggests that the $\mathrm{NiO}$ particles play the role of the catalysts for CNF growth (as indicated in Fig. S8). Clearly, based on the above results of morphology and composition, hierarchical architecture with "helixsheet-fiber-dot" structure is successfully synthesized.

The crystal and phase structure of the $\mathrm{GCA}-\mathrm{M}_{0.2}-Y$ and $\mathrm{GCA}-\mathrm{M}_{0.2}-Y(Y=0,5,10$, and 20) aerogels were characterized by XRD patterns and Raman spectra. Figure $4 a, b$ shows the XRD patterns of the samples. It is observed that the diffraction peaks of $\mathrm{GCA}-\mathrm{M}_{0.2}-0$ and GCA- $\mathrm{M}_{0.3}-0$ are in accordance with standard records of $\alpha-\mathrm{Fe}_{2} \mathrm{O}_{3}$ and $\mathrm{NiO}$ (JCPDF No. 33-0664 and 89-7130) [68, 69]. After the CVD process, the XRD diffraction peaks of GCA $-\mathrm{M}_{0.2}-Y$ and GCA- $\mathrm{M}_{0.3^{-}}-Y$ shift obviously. The typical diffraction peaks at $18.3^{\circ}, 30.6^{\circ}, 35.5^{\circ}, 43.0^{\circ}, 57.2^{\circ}$, and $62.7^{\circ}$ correspond well to the (220), (311), (400), (511), and (440) planes of $\mathrm{Fe}_{3} \mathrm{O}_{4}$ (JCPDF No. 19-0629). Meanwhile, the typical peak at $44.8^{\circ}$ corresponding to the (111) plane would be assigned to face-centered cubic Ni (JCPDF No. 04-0850 [7, 65]. Meanwhile, the wide peaks at about $25^{\circ}$ in the XRD patterns are ascribed to the existence of the carbon materials (JCPDF No. 50-0926). On the other hand, The Raman spectrometer was further employed to evaluate the carbon qualities. Figure $4 \mathrm{c}$, d exhibits the Raman spectrums of the GCA-M $\mathrm{M}_{0.2}-Y$ and GCA $-\mathrm{M}_{0.3}-Y$ aerogels, respectively. The comprehensive information is investigated by the intensity ratio of the $\mathrm{D}$ and $\mathrm{G}$ peaks of the carbon $\left(I_{\mathrm{D}} / I_{\mathrm{G}}\right)$. It is concluded that with the continuous increase in CVD reaction time, the values of $I_{\mathrm{D}} / I_{\mathrm{G}}$ decrease, which may be due to the density and length of graphited CNFs increases. This result indicates that the conductivity and conductive loss of samples could be orderly tailored by introducing 1D CNFs. Meanwhile, carbon content changes of GCA-M $\mathrm{M}_{0.3}-10$ and GCA- $\mathrm{M}_{0.2}-10$ were investigated using thermogravimetric analysis (TGA). As shown in Fig. 4e, the residual contents of $\mathrm{GCA}-\mathrm{M}_{0.3}-10$ and $\mathrm{GCA}-\mathrm{M}_{0.2}-10$ reach to 45.1 and $32.4 \mathrm{wt} \%$, respectively. This result proves that the content of carbon decreases by increasing the content of metal oxide particles. On the other hand, to clarify the changes of chemical valence states before and after the CVD process, the samples were examined by the XPS technique. As shown in Fig. 4f, the XPS full-survey-scan spectrums of GCA $-\mathrm{M}_{0.3}-0$ and $\mathrm{GCA}-\mathrm{M}_{0.3}-20$ confirm the presence of $\mathrm{C}, \mathrm{O}, \mathrm{Fe}$, and Ni elements. Furthermore, for $\mathrm{GCA}-\mathrm{M}_{0.3}-0$, the high-resolution XPS spectrum of the Ni displays two characteristic peaks at 856.2 and $873.7 \mathrm{eV}$, which are assigned to $\mathrm{Ni} 2 p_{1 / 2}$ and $2 p_{3 / 2}$ peaks of $\mathrm{Ni}^{2+}$, respectively (Fig. 4g). However, for $\mathrm{GCA}-\mathrm{M}_{0.3}-20$, the characteristic peaks centering at 853.0 and $870.2 \mathrm{eV}$ are assigned to the form of $\mathrm{Ni}^{0}$, and the rest of the peaks corresponded to the position of $\mathrm{Ni}^{2+}$ [70]. Besides, as shown in Fig. 4h, the $\mathrm{Fe}$ $2 p$ spectrum for $\mathrm{GCA}-\mathrm{M}_{0.3}-0$ mainly consists of a series of characteristic peaks of $\mathrm{Fe}^{3+}$, and the corresponding $\mathrm{Fe}$ $2 p_{3 / 2}$ peak at $711.2 \mathrm{eV}$ and the Fe $2 p_{1 / 2}$ peak at $725.1 \mathrm{eV}$ are in agreement with previously reported data [65]. For GCA- $\mathrm{M}_{0.3}-20$, the intensity of characteristic peak for $\mathrm{Fe}^{2+}$ at $710.1 \mathrm{eV}$ is significantly enhanced, which is ascribed to the formation of $\mathrm{Fe}_{3} \mathrm{O}_{4}$ [71]. The XPS results further confirm that the $\mathrm{Fe}_{2} \mathrm{O}_{3}$ and $\mathrm{NiO}$ in precursor are reduced to $\mathrm{Fe}_{3} \mathrm{O}_{4}$ and $\mathrm{Ni}$, respectively.

\subsection{Microwave Absorption Performance of As-obtained Aerogels}

Figure 5a-f gives the 3D RL curves of the as-obtained composites at different thicknesses with only $15 \%$ filling ratio. In general, the performances of EMAMs are evaluated using the optimal value of $\mathrm{RL}_{\text {min }}$ and EAB. As shown in Fig. 5a and $\mathrm{S} 9 \mathrm{a}$, the $\mathrm{RL}_{\text {min }}$ value of $\mathrm{GCA}-\mathrm{M}_{0.2}-5$ is $-22.7 \mathrm{~dB}$ at the thickness of $5.5 \mathrm{~mm}$. However, the smaller part of the EAB region suggests poor performance. Furthermore, the $\mathrm{RL}_{\text {min }}$ 

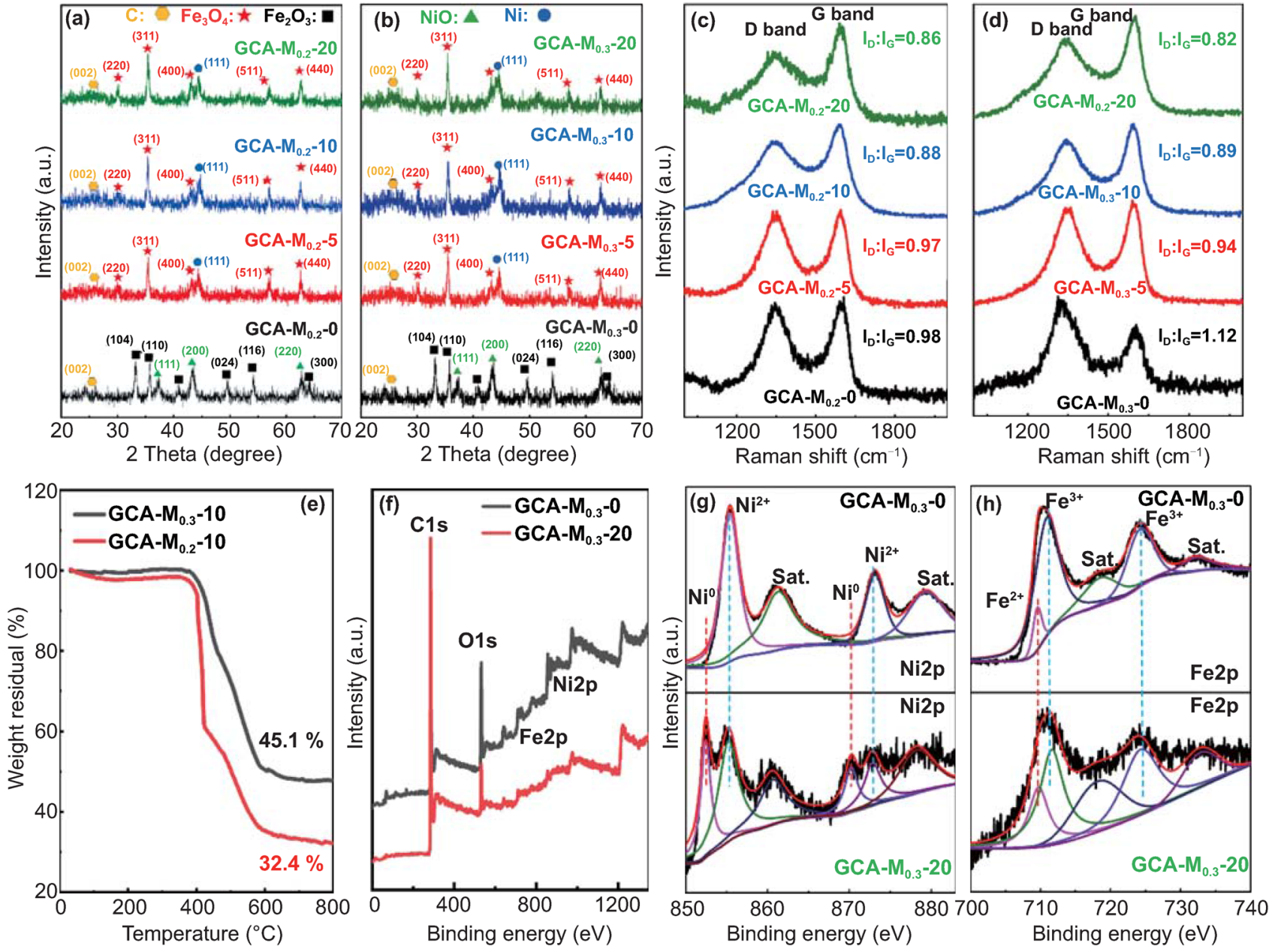

Fig. $4(\mathbf{a}, \mathbf{b})$ XRD patterns of $\mathrm{GCA}-\mathrm{M}_{0.2}-Y$ and GCA- $\mathrm{M}_{0.3}-Y$, respectively. (c, d) Raman spectra of GCA-M $\mathrm{M}_{0.2}-Y$ and GCA-M $0.3-Y$, respectively. (e) Thermogravimetric curves of GCA- $\mathrm{M}_{0.3}-10$ and GCA- $\mathrm{M}_{0.2}-10$. (f) XPS survey spectra of GCA-M $\mathrm{M}_{0.3}-0$ and GCA-M $\mathrm{M}_{0.3}-20$. (g, h) High resolution XPS spectra of Ni $2 p$ and $\mathrm{Fe} 2 p$ for GCA $-\mathrm{M}_{0.2}-\mathrm{Y}$ and $\mathrm{GCA}-\mathrm{M}_{0.3}-\mathrm{Y}$

value of GCA- $\mathrm{M}_{0.2}-10$ arrives $-55.1 \mathrm{~dB}$ at $13.8 \mathrm{GHz}$, and the corresponding $\mathrm{EAB}$ is $5 \mathrm{GHz}$ with a thickness of only $1.9 \mathrm{~mm}$. Moreover, when the thickness of absorber reduces to $1.8 \mathrm{~mm}$, the value of EAB is up to $5.6 \mathrm{GHz}$ from 12.4 to $18 \mathrm{GHz}$, almost covering the $93 \%$ of Ku band (Fig. $5 \mathrm{~b}$, $\mathrm{g}$ and $\mathrm{S} 9 \mathrm{~b})$. Besides, as displayed by Fig. $5 \mathrm{c}$ and S9c, the GCA- $\mathrm{M}_{0.2}-20$ exhibits a degraded $\mathrm{RL}_{\text {min }}$ value of only - $19.2 \mathrm{~dB}$, and the corresponding EAB exceeding - $10 \mathrm{~dB}$ is $4.8 \mathrm{GHz}$. Moreover, the microwave attenuation capacity of samples would also be adjusted by tailoring the content of M-NPs. Unfortunately, with the increase in M-NPs content, the GCA- $\mathrm{M}_{0.3}-5$ and GCA- $\mathrm{M}_{0.3}-10$ exhibit the negligible values of $\mathrm{RL}_{\min }$ and $\mathrm{EAB}$ (Fig. 5d, e and S9d, e). Surprisingly, for sample GCA-M $\mathrm{M}_{0.3}-20$ (Fig. $5 \mathrm{f}$ and S9f), it exhibits the outstanding $R L_{\min }$ value of $-71.5 \mathrm{~dB}$ at $9.5 \mathrm{GHz}$ with a thickness of $2.95 \mathrm{~mm}$. Moreover, the broaden EAB of $4.5 \mathrm{GHz}(8.2-12.7 \mathrm{GHz})$ is obtained, which covers the whole $\mathrm{X}$ band (Fig. $5 \mathrm{~g}$ ). In addition, the microwave absorbing performance of the samples before the CVD reaction $\left(\mathrm{GCA}-\mathrm{M}_{0.2}-0\right.$ and GCA- $\left.\mathrm{M}_{0.3}-0\right)$ has also been tested. Due to the weak dielectric loss and magnetic loss, their exhibit almost negligible performance (as indicated in Fig. S10). Figure 5h, i compares, in detail, the microwave absorption performances of as-obtained GCA- $\mathrm{M}_{X}-Y(X=0.2,0.3$, and $Y=5,10,20$, respectively) with thicknesses of $1.5-5.0 \mathrm{~mm}$. Clearly, it is observed that GCA- $\mathrm{M}_{0.2}-10$ and GCA $-\mathrm{M}_{0.3}-20$ exhibit outstanding performance at the relatively thin thickness. Furthermore, we compared the microwave absorption 

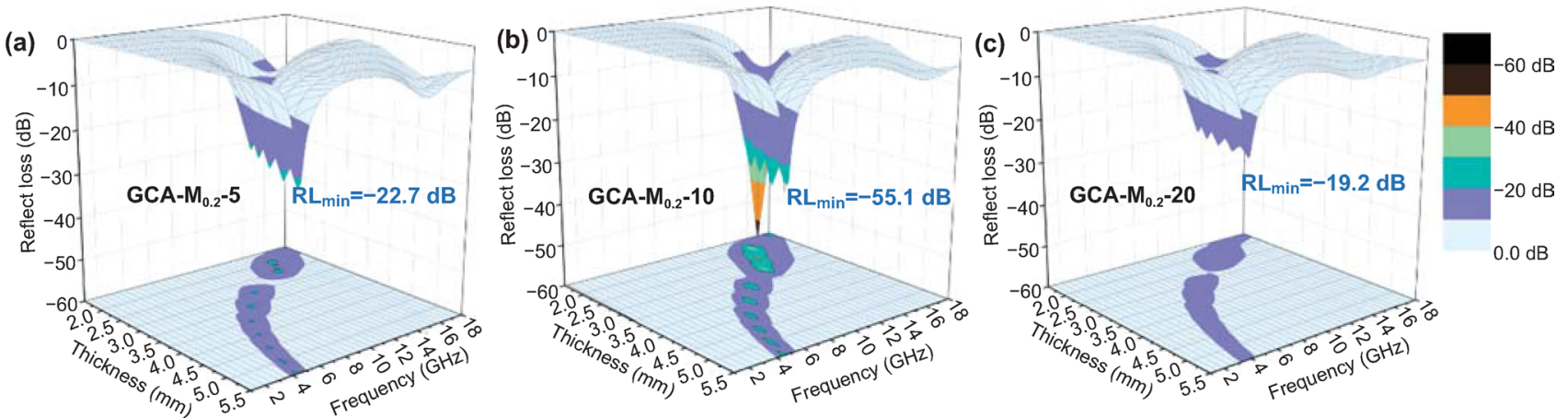
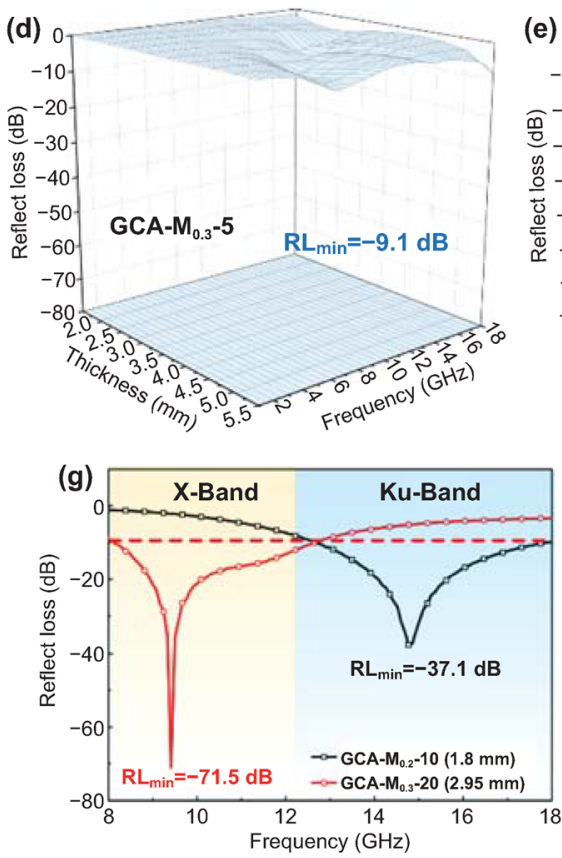
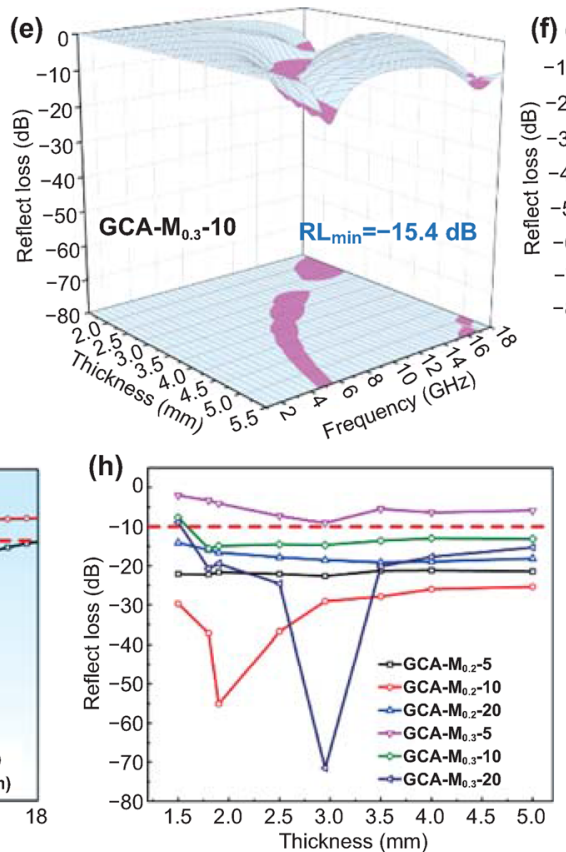
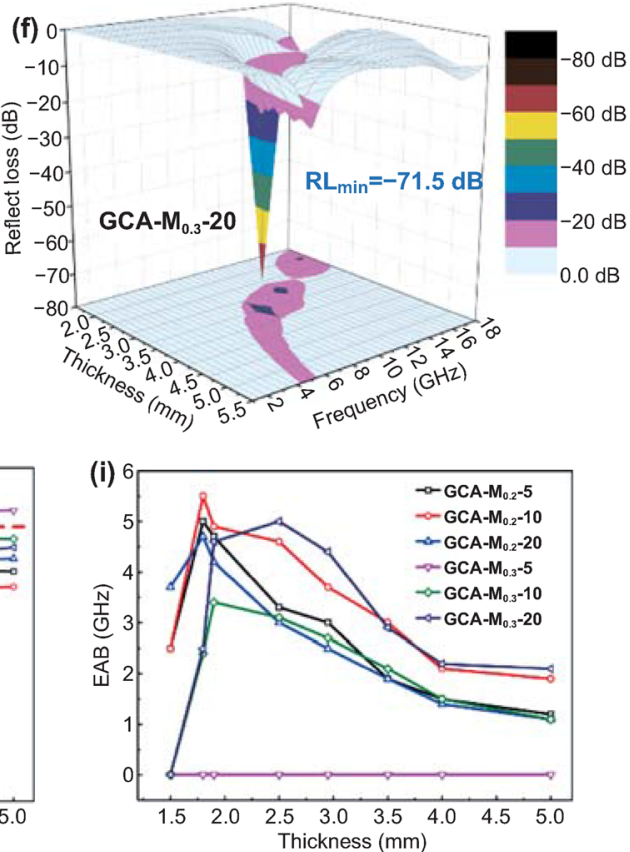
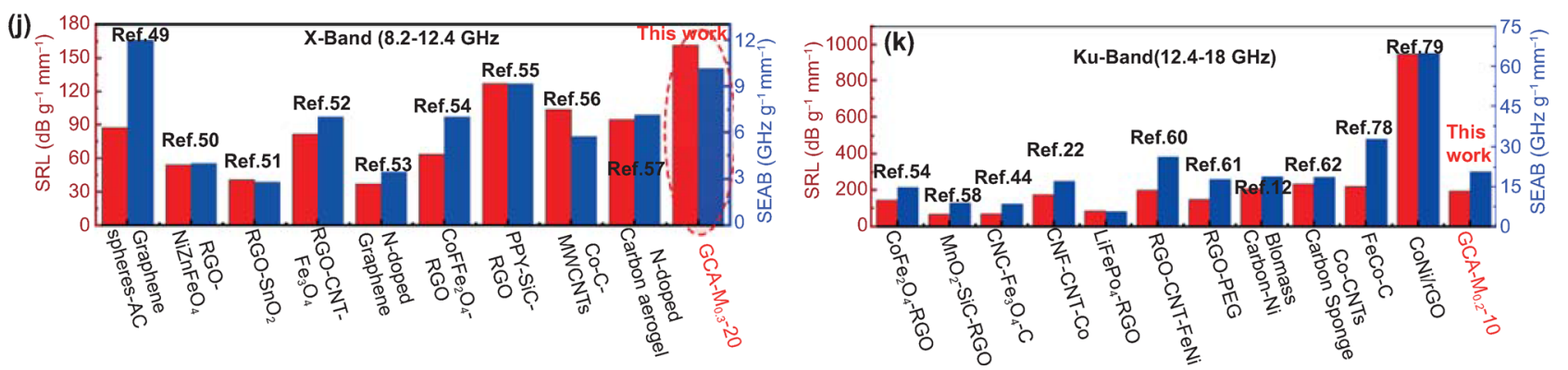

Fig. 5 3D representations of the RL of (a) GCA-M $\mathrm{M}_{0.2}-5$, (b) GCA- $\mathrm{M}_{0.2}-10$, (c) GCA- $\mathrm{M}_{0.2}-20$, (d) GCA-M 0.3 , (e) GCA-M 0.3 , and (f) GCA-M $\mathrm{M}_{0.3}-20$. (g) Maximum EABs of the GCA-M $\mathrm{M}_{0.2}-10$ and $\mathrm{GCA}-\mathrm{M}_{0.3}-10$ aerogels. (h, i) $\mathrm{RL}_{\text {min }}$ and EAB values of as-obtained samples with different thicknesses, respectively. (j, k) Comparison of specific RL and specific EAB values of recently reported carbon-based aerogels in Xand Ku-band

performances of GCA- $\mathrm{M}_{0.2}-10$ and GCA- $\mathrm{M}_{0.3}-20$ with the state-of-the-art carbon-based absorbers (published during the last 3 years). For the comprehensive comparison regarding performances of the as-mentioned EMAMs, the specific RL $\left(\mathrm{SRL}, \mathrm{RL}_{\text {min }} /(\right.$ thickness $\times$ filler loading)) and specific $\mathrm{EAB}$
(SEAB, $\mathrm{EAB} /($ thickness $\times$ filler loading)) values are calculated and given by Fig. $5 \mathrm{j}$ and k, respectively. Among all, The GCA- $\mathrm{M}_{0.3}-20$ fabricated in this work exhibits a superior SRL value $\left(161.5 \mathrm{~dB} \mathrm{~mm}^{-1} \mathrm{~g}^{-1}\right)$ in $\mathrm{X}$-band, and the SEAB value of GCA-M $\mathrm{M}_{0.3}-20\left(10.16 \mathrm{GHz} \mathrm{mm} \mathrm{m}^{-1} \mathrm{~g}^{-1}\right)$ is also relative 
superior to other listed EMAMs (Fig. 5j). Besides, it is clear from Fig. $5 \mathrm{k}$ that the SRL and SEAB values of GCA-M $\mathrm{M}_{0.2}-10$ exhibit advantages by overall consideration of absorber loading $(<20 \mathrm{wt} \%)$ and thickness $(<2 \mathrm{~mm})$ in Ku-band. On the other hand, the corresponding tables (Tables S2 and S3) including each works from the literatures, not only the RL value, but also the frequency range, the typical thickness, and the filling ratio of the samples are summarized in supplementary materials. According to the above comparison, it is concluded that the as-obtained hierarchical aerogels in this paper would act as an extremely promising candidate for a lightweight, thin, and highly efficient MWAMs.

\subsection{Electromagnetic Parameters of As-obtained Aerogels}

The dependence of the complex permittivity $\left(\varepsilon_{\mathrm{r}}=\varepsilon_{\mathrm{r}}^{\prime}-\varepsilon_{\mathrm{r}}^{\prime \prime}\right)$ and complex permeability $\left(\mu_{\mathrm{r}}=\mu_{\mathrm{r}}^{\prime}-\mu_{\mathrm{r}}^{\prime \prime}\right)$ on frequency from 1 to $18 \mathrm{GHz}$ was further discussed for understanding the microwave absorbing mechanism of as-obtained aerogels, and the results were given by Fig. 6. All in all, the real parts $\left(\varepsilon_{\mathrm{r}}^{\prime}\right.$ and $\left.\mu_{\mathrm{r}}^{\prime}\right)$ of the electromagnetic parameters represent the storage capability of electromagnetic wave energy, and the imaginary parts $\left(\varepsilon_{\mathrm{r}}^{\prime \prime}\right.$ and $\left.\mu_{\mathrm{r}}^{\prime \prime}\right)$ stand for the ability of energy dissipation [9]. It is observed that all the $\varepsilon_{\mathrm{r}}^{\prime}$ values exhibit a decreasing tendency in the frequency range of 1 to $12 \mathrm{GHz}$, then display the relative constant values in $12-18 \mathrm{GHz}$ (Fig. 6a). Meanwhile, as shown in Fig. $6 \mathrm{~b}$, three remarkable vibration peaks are observed at $\sim 8.8 \mathrm{GHz}$ (labeled as A), $10.5 \mathrm{GHz}$ (labeled as B), and $\sim 13.2 \mathrm{GHz}$ (labeled as $\mathrm{C}$ ), which suggest relaxation behavior exist in $\varepsilon_{\mathrm{r}}^{\prime \prime}$. Considering the dimensional gradient of as-prepared samples, it is reasonable that the local space charge accumulation of heterojunction interface and the surface geometry induce enhancement effect for dielectric loss [16]. Besides, the dielectric loss tangent $\tan \delta_{\mathrm{e}}$ values were computed to evaluate dielectric dissipation capability. The order of $\tan \delta_{\mathrm{e}}$ values for $\mathrm{GCA}-\mathrm{M}_{X}-Y$ samples is GCA $-\mathrm{M}_{X}-20>$ GCA $-\mathrm{M}_{X}-10>\mathrm{GCA}^{-} \mathrm{M}_{X}-5(X=0.2,0.3)$, which gives the evidence that the introduction of 1D CNFs is an effective strategy to boost the dielectric attenuation capacity. Meanwhile, it should be noted that the conductive paths composed of the 1D structures are the key to improving the dielectric loss. To solidate this viewpoint, the electromagnetic parameters of GCA- $\mathrm{M}_{0.2}-0$ and GCA- $\mathrm{M}_{0.3}-0$ were tested and compared with those of GCA- $\mathrm{M}_{0.2}-10$ and GCA- $\mathrm{M}_{0.3}-20$, respectively. After the introduction of CNFs (as shown in Fig. S11a, b), the imaginary part of complex permittivity is significantly increased, which suggests the
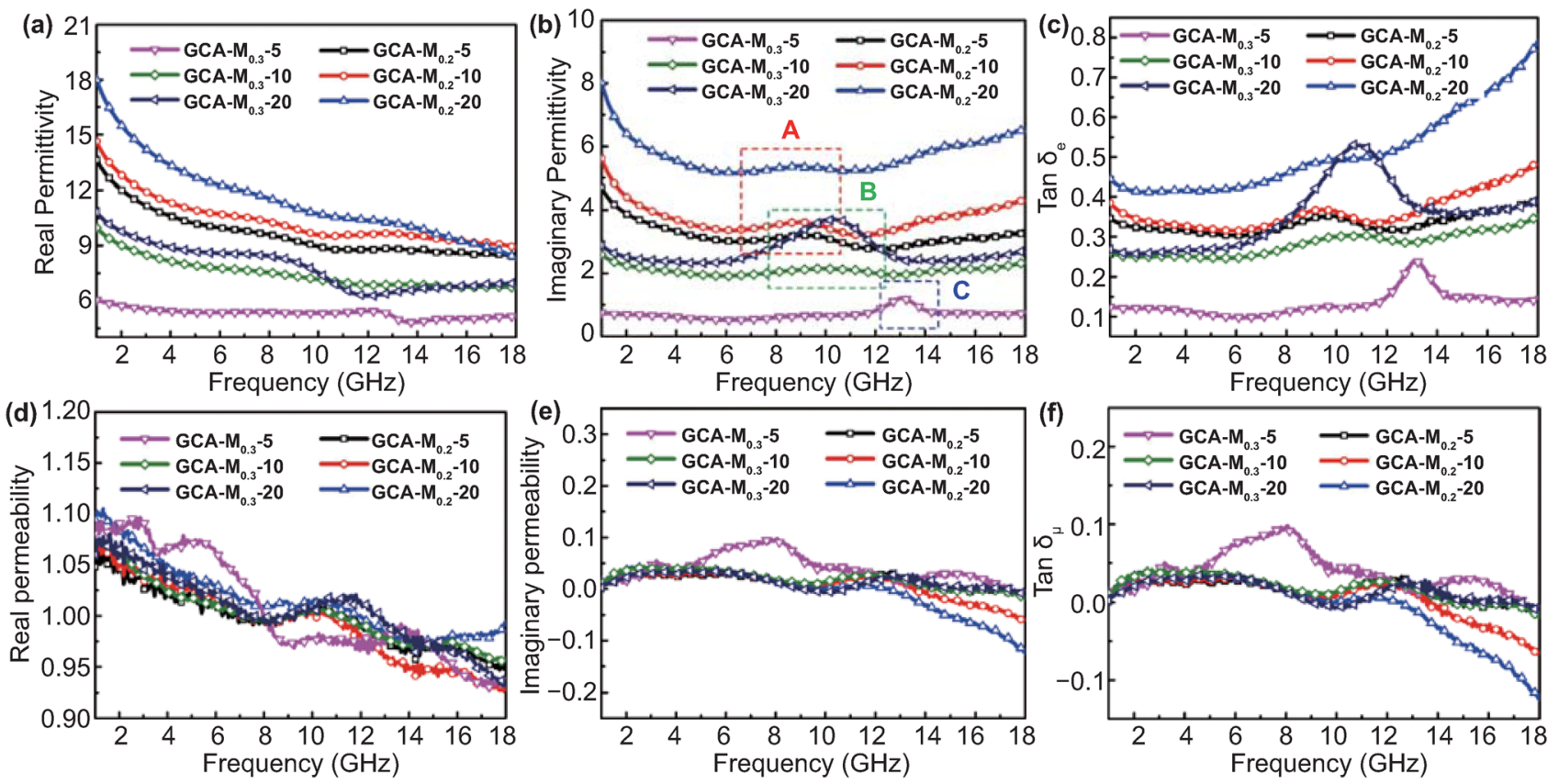

Fig. 6 Frequency dependence of (a) real permittivity, (b) imaginary permittivity, (c) dielectric loss tangent, (d) real permeability, (e) imaginary permeability, and (f) magnetic loss tangent 
improvement of dielectric loss (as indicated by Fig. S11c, d). Moreover, the dielectric loss mechanism is analyzed by the relationship between $\varepsilon_{\mathrm{r}}^{\prime}$ and $\varepsilon_{\mathrm{r}}^{\prime \prime}$. Based on the Debye relaxation theory, if the curve of $\varepsilon_{\mathrm{r}}^{\prime}$ versus $\varepsilon_{\mathrm{r}}^{\prime \prime}$ exhibits semicircle morphology, these MWAMs would possess strong polarization behavior, and each semicircle is denoted as Cole-Cole semicircle [29]. For GCA- $\mathrm{M}_{0.2}-Y$ samples, except for the two semicircles, the corresponding curves also possess a distinguishable straight line in the tail, suggesting that the high conductive loss originating from the conductive 3D carbon network in the MWAMs/wax composites (Fig. $\mathrm{S} 12 \mathrm{a}-\mathrm{c})$. Meanwhile, GCA-M $0.3 \mathrm{Y}$ samples exhibit three Cole-Cole semicircles and shortened "line tail", indicating the enhanced polarization relaxation and relatively low conductive loss [2]. These results mentioned above reveal that the dielectric loss of GCA- $\mathrm{M}_{\mathrm{X}}-\mathrm{Y}$ originates approximatively from relaxation loss and conductive loss. Besides, Fig. 6d-f reveal the complex permeability of the as-prepared aerogels. Briefly, the real $\left(\mu_{\mathrm{r}}^{\prime}\right)$ and imaginary $\left(\mu_{\mathrm{r}}^{\prime \prime}\right)$ parts of all the samples are almost near 1 and 0 , respectively, suggesting the magnetic loss is relatively weak due to the inconspicuous natural magnetic resonance (Fig. 6e) and eddy current loss (Fig. S13a). Meanwhile, the negligible tan $\delta$ values of as-obtained aerogels also prove this viewpoint (Fig. 6f). Even so, the presence of magnetic loss would ameliorate the impedance match and boost the dissipation of electromagnetic energy. Moreover, the manipulated $\mu_{\mathrm{r}}^{\prime}$ and $\mu_{\mathrm{r}}^{\prime \prime}$ values could induce the absorption peaks to transfer to a lower frequency.

In general, the ideal MWAMs should possess powerful electromagnetic wave dissipation ability and excellent impedance matching synchronously. Normally the attenuation constant $(\alpha)$ is used for the analysis of the inherent attenuation ability and calculated by Eq. (3) [44]: performance must be taken into account of microwave absorption process [72-77]. Based on previous research, when the $Z$ value is equal or close to 1 , the incident electromagnetic wave would easily enter the MWAM and then be converted to other forms of energy. The $Z$ values of GCA- $\mathrm{M}_{X}-Y$ aerogels at different thicknesses are computed and displayed in Fig. $7 \mathrm{a}-\mathrm{f}$. It can be seen that GCA-M $\mathrm{M}_{0.2}-10$ has good impedance matching performance in almost the entire frequency range, which is derived from appropriate conductive loss and corresponding magnetic loss [78-81]. As a comparison, the $Z$ value of the GCA- $\mathrm{M}_{0.3}-5$ is far from 1 , and most of them are over 1.6 in the whole frequency range, suggesting the poorest impedance matching performance among all the samples. Linking back to the poor $\tan \delta_{\mathrm{e}}$ and non-negligible $\tan \delta_{\mathrm{e}}$ values for GCA- $\mathrm{M}_{0.3}-5$, it is reasonable that the matched dielectric and magnetic loss are two prerequisites for as-prepared aerogels to achieve the outstanding impedance matching performance. Furthermore, take GCA- $\mathrm{M}_{0.2}-10$ and GCA- $\mathrm{M}_{0.3}-20$ as the examples, the relationship between the $\mathrm{RL}_{\text {min }}, \alpha$, and $Z$ values is analyzed and given by Fig. $7 \mathrm{~g}$, f, respectively. It is clearly seen that as $Z$ value is equal to 1 , the RL values arrived at the minimum at 13.8 and $9.5 \mathrm{GHz}$ for $\mathrm{GCA}-\mathrm{M}_{0.2}-10$ and $\mathrm{GCA}-\mathrm{M}_{0.3}-20$, respectively. Furthermore, we select GCA- $\mathrm{M}_{0.2}-10$ as an illustration to analyze the effort of $\alpha$ and $Z$ on RL value. As shown in Fig. $7 \mathrm{~g}$, two points reach 1 in the $Z$ curve, which are noted as $i$ and $i i$. It is seen that point $i i$ exhibits better microwave absorption performance than that of point $i$, suggesting larger $\alpha$ would boost $\mathrm{RL}_{\text {min }}$ value under the same $Z$ value. All these results discussed above certify that the stronger attenuation ability with excellent impedance matching is a key point for our aerogel as an ideal MWAM.

\subsection{Roles of CNC}

In order to clarify the key functions of the $\mathrm{CNC}$ in the system, we prepared two control aerogels without $\mathrm{CNC}$, and

$\alpha=\frac{\sqrt{2} \pi f}{c} \sqrt{\left(\mu_{\mathrm{r}}^{\prime \prime} \varepsilon_{\mathrm{r}}^{\prime \prime}-\mu_{\mathrm{r}}^{\prime \prime} \varepsilon_{\mathrm{r}}^{\prime \prime}\right)+\sqrt{\left(\mu_{\mathrm{r}}^{\prime \prime} \varepsilon_{\mathrm{r}}^{\prime \prime}-\mu_{\mathrm{r}}^{\prime \prime} \varepsilon_{\mathrm{r}}^{\prime \prime}\right)^{2}+\left(\mu_{\mathrm{r}}^{\prime \prime} \varepsilon_{\mathrm{r}}^{\prime \prime}+\mu_{\mathrm{r}}^{\prime \prime} \varepsilon_{\mathrm{r}}^{\prime \prime}\right)^{2}}}$

It is observed that all the $\alpha$ values increase with elevating the frequency and approximatively decrease with increasing M-NPs content (Fig. S13b). Furthermore, GCA-M $\mathrm{M}_{0.2}-20$ shows the largest $\alpha$ value in the whole test frequency range. However, owing to the superfluous dielectric loss of GCA-M $\mathrm{M}_{0.2}-20$, seriously impedance mismatching occurs and results in a poor microwave absorption performance. Therefore, the impedance matching $\left(\mathrm{Z}=\left|\mathrm{Z}_{\text {in }} / \mathrm{Z}_{0}\right|\right)$ the corresponding samples were labeled as $\mathrm{GA}-\mathrm{M}_{0.2}-10$ (Fig. S5a) and GA-M $\mathrm{M}_{0.3}-20$ (Fig. S5b), respectively. Typically, the dielectric performance of MWAMs is highly related to their electrical conductivity, and Fig. 8a gives the measured conductivities of as-prepared aerogel/wax composites. The electrical conductivities of GCA- $\mathrm{M}_{0.2}-10$-, GCA- $\mathrm{M}_{0.3}-20-$, GA- $\mathrm{M}_{0.2}-10-$, and GCA- $\mathrm{M}_{0.3}-20$-wax are 

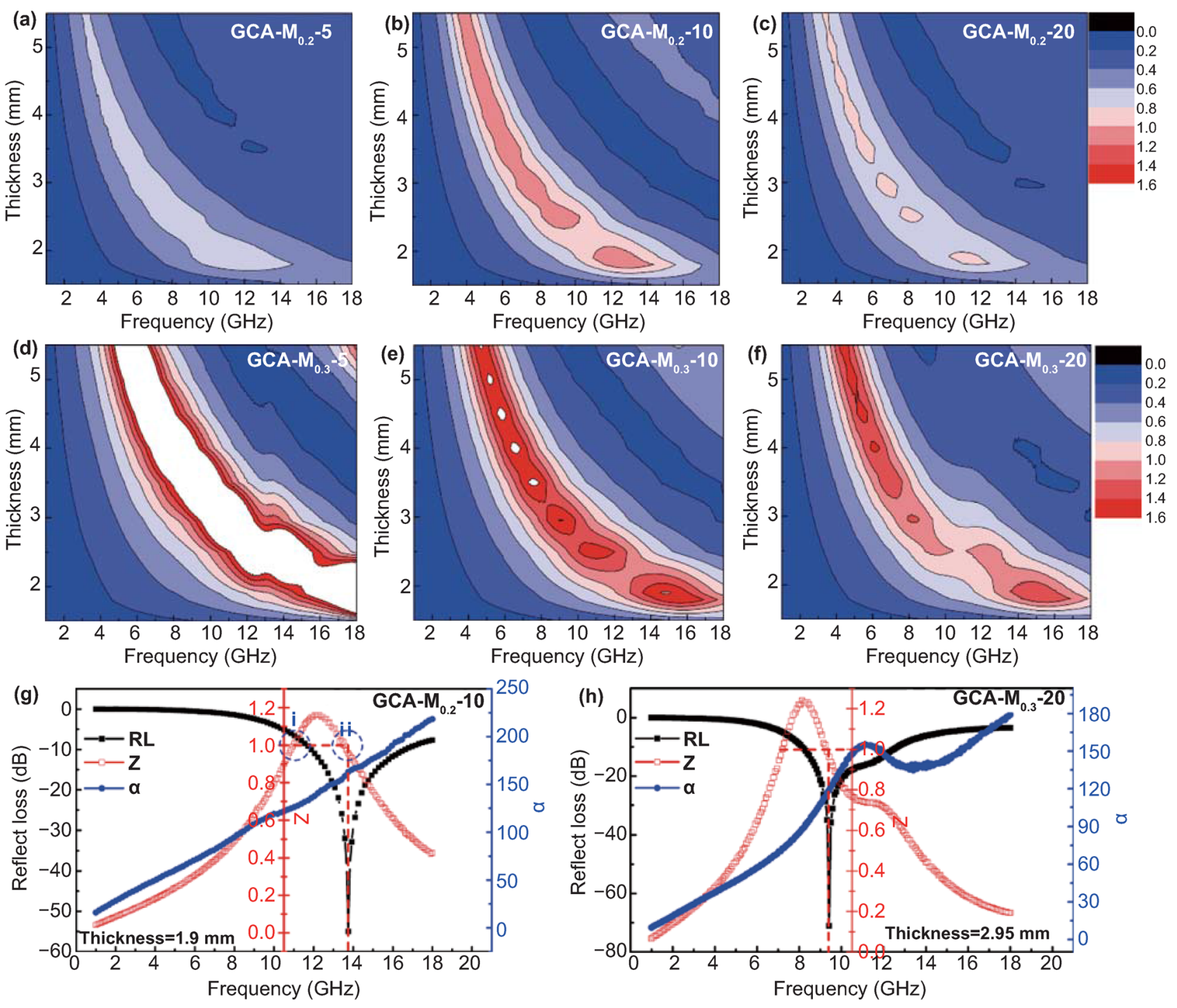

Fig. 7 Calculated $Z$ values at different thicknesses from 1 to $18 \mathrm{GHz}$ for (a) $\mathrm{GCA}-\mathrm{M}_{0,2}-5$, (b) GCA-M $0.2-10$, (c) GCA-M $\mathrm{M}_{02}-20$, (d) GCA$\mathrm{M}_{0.3}-5$, (e) GCA- $\mathrm{M}_{0.3}-10$, and (f) GCA- $\mathrm{M}_{0.3}-20$. Frequency-dependent RL, $\alpha$, and $Z$ values for (g) GCA-M $0.2-10$ and (h) GCA-M $0.3-20$

$1.58 \times 10^{-3}, 0.89 \times 10^{-3}, 1.65 \times 10^{-2}$, and $1.17 \times 10^{-2} \mathrm{~S} \mathrm{~m}^{-1}$, respectively. It is concluded that the electrical conductivity obviously degrades with adding the CNCs in aerogel, and the measured $I-V$ curves (Fig. 8b, c) are also consistent with this viewpoint. At present, most researchists have agreed that the conductivity of the sample is closely related to the crystal structure and morphology of materials [82-86]. In this case, the crystal structure of $\mathrm{CNC}$ is almost amorphous (Fig. 3e), which indicates the conductivity of the CNC lower than that of graphene and CNF. More importantly, as shown in Figs. 8d, e, and S14, the helical CNC are inserted graphene layers, resulting in the formation of point-to-surface contact (CNC-graphene) instead of surface-to-surface contact (graphene-graphene). Therefore, the evolution from surface-tosurface contact to point-to-surface contact leads to a decrease in electrical conductivity. Besides, the existence of helical CNCs would create the gaps and pores between the graphene layers, which lengthen the transmission distances of microwave owing to the multiple reflect and scatter effects. Moreover, based on the Maxwell-Garnett theory, the abundant void 

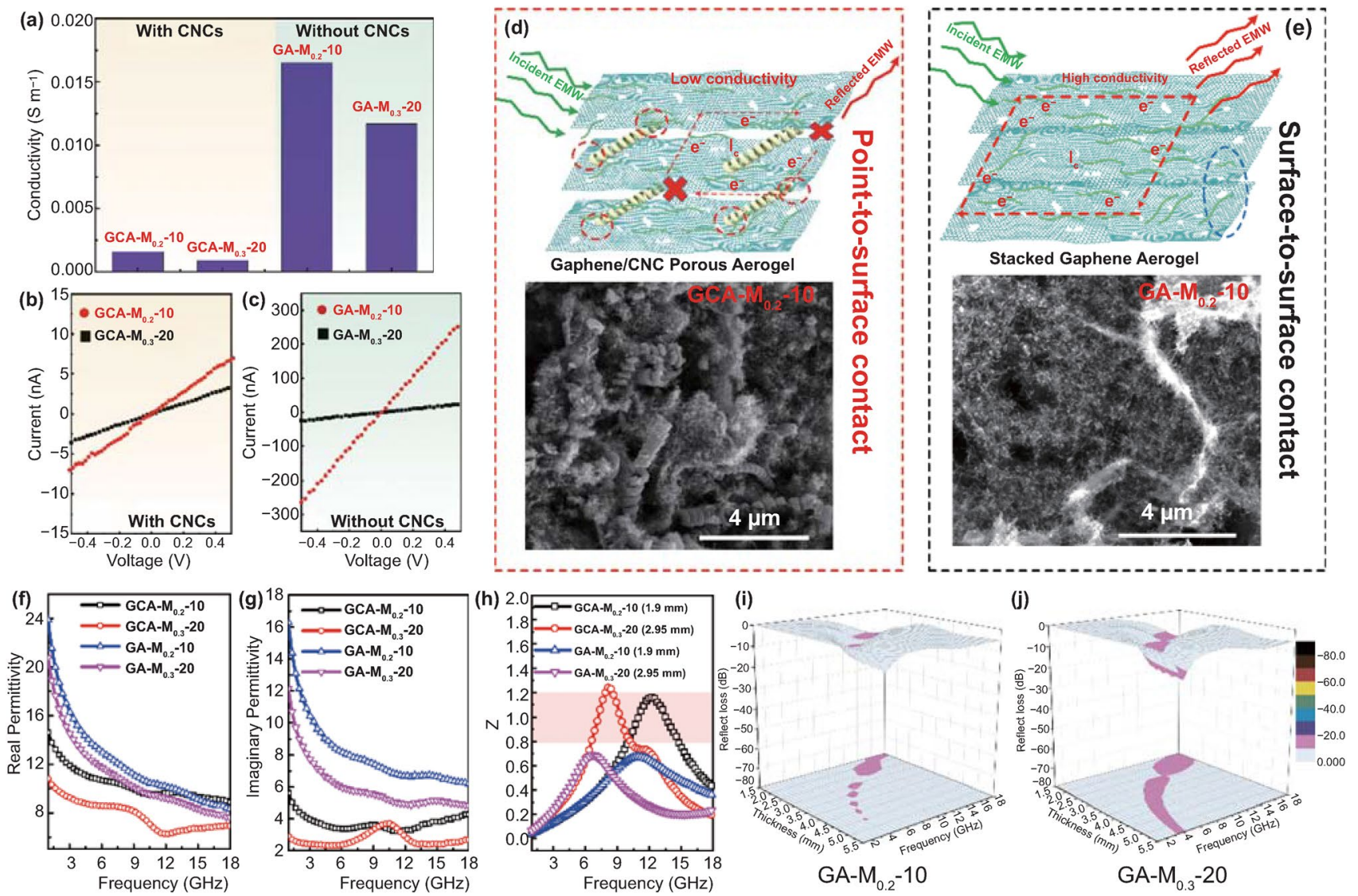

Fig. 8 (a) Electrical conductivities of GCA- $\mathrm{M}_{0.2}-10-$, GCA- $\mathrm{M}_{0.3}-20-$, GA- $\mathrm{M}_{0.2}-10-$, and GCA- $\mathrm{M}_{0.3}-20$-wax composites. (b) Measured $I-V$ curves of GCA- $\mathrm{M}_{0.2}-10$ - and GCA- $\mathrm{M}_{0.3}-10$-wax composites. (c) Measured $I-V$ curves of GA- $\mathrm{M}_{0.2}-10$ - and GA-M $0.3-20$-wax composites. (d, e) Schematic of structure-function effects of CNC on dielectric property of as-obtained aerogels. (f) Real permittivity, (g) imaginary permittivity, and (h) $Z$ values for GCA- $\mathrm{M}_{0.2}-10-$, GCA- $\mathrm{M}_{0.3}-20-$, GA- $\mathrm{M}_{0.2}-10-$, and GA- $\mathrm{M}_{0.3}-20$-wax composites

spaces of as-obtained hierarchical aerogels would effectively reduce the complex permittivity, thus improving impedance matching [52]. As a result, the dielectric property would change dramatically. To further shed light on the effect of the CNC skeleton in an aerogel, electromagnetic parameters of the GA- $\mathrm{M}_{0.2}-10$ and $\mathrm{GA}-\mathrm{M}_{0.3}-20$ were measured and compared, as shown in Fig. 8f, g, and S10. For a comparison purpose, we also give the electromagnetic parameters of the GCA- $\mathrm{M}_{0.2}-10$ and GCA- $\mathrm{M}_{0.3}-20$. As shown in Fig. S15, the complex permeability of the GCA- $\mathrm{M}_{X}-Y$ aerogels is similar to that of $\mathrm{GA}-\mathrm{M}_{X}-Y$ aerogels. This result suggests that the effect of CNCs on permeability is negligible. Besides, the $\varepsilon_{\mathrm{r}}^{\prime}$ and $\varepsilon_{\mathrm{r}}^{\prime \prime}$ values of $\mathrm{GA}-\mathrm{M}_{X}-Y$ samples are obviously higher than those of GCA- $\mathrm{M}_{X}-\mathrm{Y}$ samples, suggesting the aerogels without $\mathrm{CNCs}$ possess better dielectric dissipation capability in the test frequency range. Furthermore, the attenuation constants of typical GA- $\mathrm{M}_{X}-Y$ and GCA- $\mathrm{M}_{X}-Y$ samples are shown in Fig. S16. It is noted that the $\alpha$ of $\mathrm{GA}-\mathrm{M}_{X}-Y$ is higher than those of other GCA- $\mathrm{M}_{X}-Y$ samples in the almost whole frequency range, which could be owed to the significantly enhanced conductive loss. However, the impedance matching of GCA- $\mathrm{M}_{X}-Y$ aerogel seems to be much better than that of GA-M $\mathrm{M}_{X}-Y$ sample, which further proves the crucial role of CNC. As a result, the $\mathrm{GA}-\mathrm{M}_{X}-Y$ samples exhibit the poor performances of $\mathrm{RL}_{\text {min }}$ and EAB (Fig. 8i, j). Based on the above results, accounting for the effects arising from the amorphous structure of $\mathrm{CNC}$, point-to-surface contact between $\mathrm{CNC}$ and graphene, and the concomitant porosity, the CNC-contained aerogels eventually exhibited a significantly improved impedance matching and microwave absorption performance. 


\subsection{Microwave Absorption Mechanism}

The superior microwave absorption performance of the as-prepared aerogel is closely related to the multiple loss mechanisms of multi-dimensional gradient structures and the synergistic effects of these components. In view of these facts, the microwave absorption mechanism is graphically summarized in Fig. 9. Firstly, the detailed loss mechanisms of these components in aerogels are analyzed as following. In general, there are six factors that dominate the microwave attenuation, including conductive loss, cross-polarization, dipole polarization, multiple scattering and reflection, interfacial polarization, and magnetic loss.

For 3D structured CNC: On the one hand, CNCs would induce cross-polarization loss due to their chiral structures during the interaction with electromagnetic wave. Besides, as one of the framework materials, conductive CNCs play an important role in the formation of the conductive network in the aerogel.

For 2D structured RGO: When the electromagnetic wave transmits into the absorber, it would be repeatedly scattered by the RGO nanosheets and thus boosts the multiple scattering loss. On the other hand, the RGO nanosheets have a large number of defects, oxygen-containing functional groups, and dangling bonds, which provide active sites for dipole polarization loss.

For 1D structured CNF: In our case, CNFs with controllable length and density would construct the tailorable conductive networks. As a result, the increasing conductive networks would effectively improve the conductive loss of the material.

For 0D structured particle: The heterogeneous interface between $\mathrm{Fe}_{3} \mathrm{O}_{4}$ nanoparticle and carbon shell are benefit to the interfacial polarization, resulting in the improvement of the dielectric loss. In addition, it should be noted that

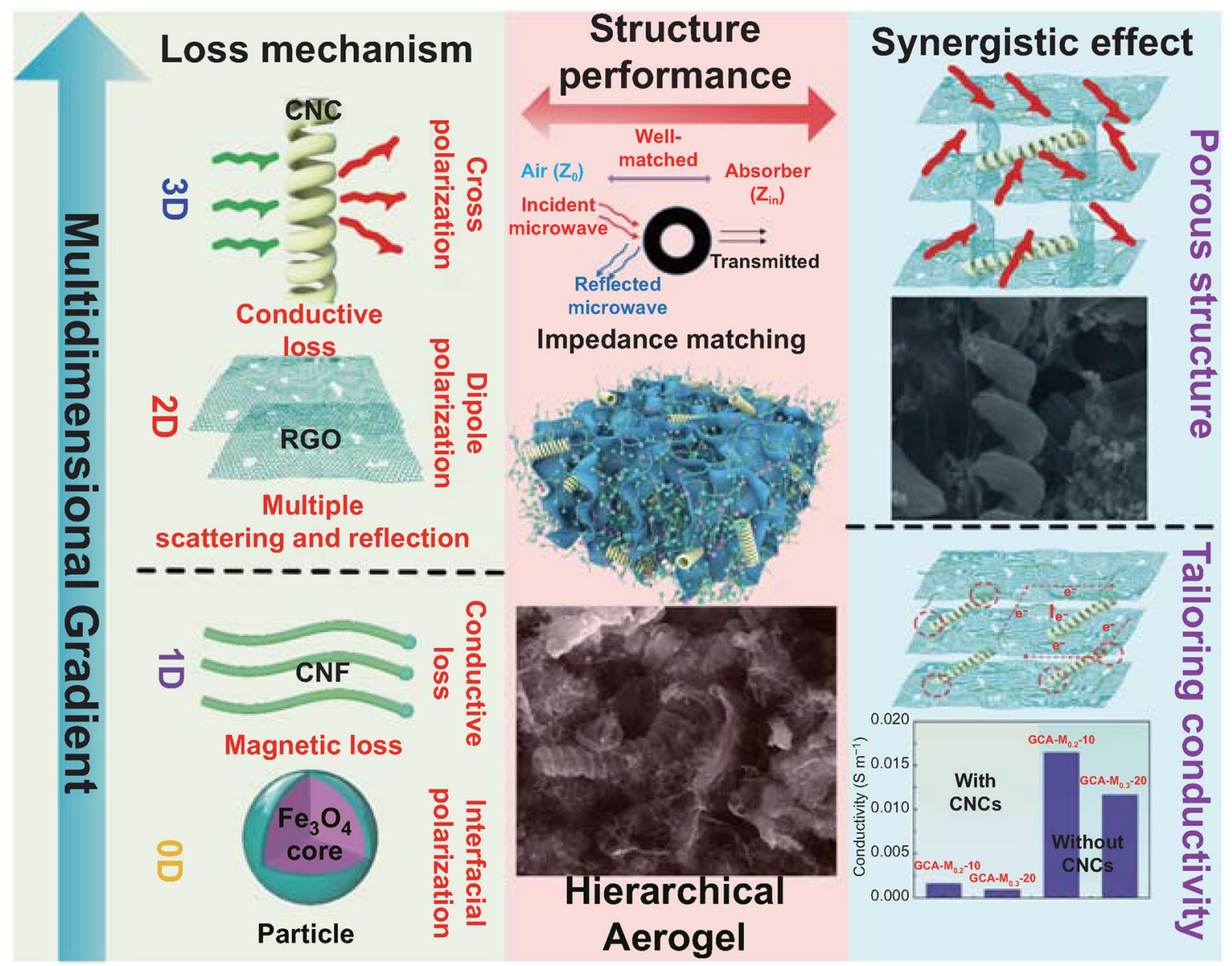

Fig. 9 Pictorial description of relative microwave loss mechanisms existing in the $\mathrm{GCA}-\mathrm{M}_{X}-Y$-wax composites 
the introduction of $\mathrm{Ni} @ \mathrm{CNF}$ and $\mathrm{Fe}_{3} \mathrm{O}_{4} @ \mathrm{C}$ would not only regulate the dielectric loss, but also offer a certain magnetic loss, leading to the improvement of the impedance matching.

Furthermore, the synergistic effects of these components are not only conducive to improving the microwave attenuation of the material, but also significantly adjust the impedance matching. Frist, the existence of helical CNCs would create the gaps and pores between the graphene layers, which lengthen the transmission distances of electromagnetic wave owing to the multiple reflect and scatter effects. Meanwhile, porous structure of aerogel ensures the growth space for CNFs, and facilitates the storage of air which would improve the impedance matching. Second, the absorbers with higher conductivity would lead to poor microwave absorption performance due to the skin effect. In this paper, the CNCs and CNFs are used to tailor the conductivity of the material. On the one hand, the helical CNCs with amorphous structures insert into graphene layers, resulting in the formation of point-to-surface contacts (CNCgraphene) instead of surface-to-surface contacts (graphene -graphene). Therefore, the evolution of contact form leads to the conductivity decrease. On the other hand, the conductivity of hierarchical aerogels is effectively controlled by tailoring the growth density and length of CNFs. As a result, the optimized aerogel has tailoring microwave absorption capacity and good impedance matching performance in almost the entire frequency range.

\section{Conclusion}

In summary, the "3D helix-2D sheet-1D fiber-0D dot" hierarchical aerogels have been successfully synthesized by sequential processes of hydrothermal self-assembly and insitu CVD growth method. In particular, the 2D graphene layers are uniformly intercalated by 3D helical CNCs, which endow the aerogel with abundant porous structure and better dielectric properties. Meanwhile, the CNCs and CNFs are successfully used to tailor the conductivity of the material. Besides, by adjusting the content of the magnetic particle and CVD reaction time, tunable electromagnetic parameters and excellent impedance matching, which play a crucial effect in the microwave absorption performance. The hierarchical GCA- $\mathrm{M}_{0.2}-10$ aerogel shows outstanding $\mathrm{RL}_{\text {min }}(-55.1 \mathrm{~dB})$ and width expansion $\mathrm{EAB}$ values $(5.6 \mathrm{GHz})$ when the mass load in the wax matrix is only
$15 \mathrm{wt} \%$. Moreover, the $\mathrm{RL}_{\min }$ value of $\mathrm{GCA}-\mathrm{M}_{0.3}-20$ aerogel reaches $-71.5 \mathrm{~dB}$ at $9.5 \mathrm{GHz}$, and the corresponding EAB covers the whole X-band. The remarkable microwave absorption performance of the as-prepared aerogel is closely related to the multiple loss mechanisms of multi-dimensional gradient structures and the synergistic effect of each component. Our findings provide valuable guidance and inspiration for the design of multilevel hierarchical structure materials for microwave absorbing application and other related fields.

Acknowledgements This work was financially supported by the National Natural Science Foundation of China (Nos. 51972039, 51803018, and 51661145025) and LiaoNing Revitalization Talents Program (No. XLYC1902122).

Open Access This article is licensed under a Creative Commons Attribution 4.0 International License, which permits use, sharing, adaptation, distribution and reproduction in any medium or format, as long as you give appropriate credit to the original author(s) and the source, provide a link to the Creative Commons licence, and indicate if changes were made. The images or other third party material in this article are included in the article's Creative Commons licence, unless indicated otherwise in a credit line to the material. If material is not included in the article's Creative Commons licence and your intended use is not permitted by statutory regulation or exceeds the permitted use, you will need to obtain permission directly from the copyright holder. To view a copy of this licence, visit http://creativecommons.org/licenses/by/4.0/.

Supplementary Information The online version contains supplementary material available at https://doi.org/10.1007/ s40820-021-00667-7.

\section{References}

1. X. Zeng, X. Cheng, R. Yu, G.D. Stucky, Electromagnetic microwave absorption theory and recent achievements in microwave absorbers. Carbon 168, 606-623 (2020). https:// doi.org/10.1016/j.carbon.2020.07.028

2. D. Zhi, T. Li, J. Li, H. Ren, F. Meng, A review of threedimensional graphene-based aerogels: synthesis, structure and application for microwave absorption. Compos. Pt. B-Eng. 211, 108642 (2021). https://doi.org/10.1016/j.compo sitesb.2021.108642

3. Q. Song, F. Ye, L. Kong, Q. Shen, L. Han et al., Graphene and MXene nanomaterials: toward high-performance electromagnetic wave absorption in gigahertz band range. Adv. Funct. Mater. 30(31), 2000475 (2020). https://doi.org/10. 1002/adfm.202000475

4. M. Zhang, C. Han, W.-Q. Cao, M.-S. Cao, H.-J. Yang et al., A nano-micro engineering nanofiber for electromagnetic absorber, green shielding and sensor. 
Nano-Micro Lett. 13(1), 27 (2021). https://doi.org/10.1007/ s40820-020-00552-9

5. G. Sun, B. Dong, M. Cao, B. Wei, C. Hu, Hierarchical dendrite-like magnetic materials of $\mathrm{Fe}_{3} \mathrm{O}_{4}, \gamma-\mathrm{Fe}_{2} \mathrm{O}_{3}$, and $\mathrm{Fe}$ with high performance of microwave absorption. Chem. Mater. 23(6), 1587-1593 (2011). https://doi.org/10.1021/cm103441u

6. J. Deng, X. Zhang, B. Zhao, Z. Bai, S. Wen et al., Fluffy microrods to heighten the microwave absorption properties through tuning the electronic state of $\mathrm{Co} / \mathrm{CoO}$. J. Mater. Chem. C 6(26), 7128-7140 (2018). https://doi.org/10.1039/C8TC0 $2520 \mathrm{G}$

7. D. Liu, Y. Du, Z. Li, Y. Wang, P. Xu et al., Facile synthesis of 3D flower-like Ni microspheres with enhanced microwave absorption properties. J. Mater. Chem. C 6(36), 9615-9623 (2018). https://doi.org/10.1039/C8TC02931H

8. Y. Duan, Z. Xiao, X. Yan, Z. Gao, Y. Tang et al., Enhanced electromagnetic microwave absorption property of peapodlike $\mathrm{MnO} @$ carbon nanowires. ACS Appl. Mater. Interfaces 10(46), 40078-40087 (2018). https://doi.org/10.1021/acsami. 8 b11395

9. J. Yang, W. Yang, F. Li, Y. Yang, Research and development of high-performance new microwave absorbers based on rare earth transition metal compounds: a review. J. Magn. Magn. Mater. 497, 165961 (2020). https://doi.org/10.1016/j.jmmm. 2019.165961

10. Y. Cheng, G. Ji, Z. Li, H. Lv, W. Liu, Facile synthesis of FeCo alloys with excellent microwave absorption in the whole $\mathrm{Ku}-$ band: effect of $\mathrm{Fe} / \mathrm{Co}$ atomic ratio. J. Alloys Compd. 704, 289-295 (2017). https://doi.org/10.1016/j.jallcom.2017.02.024

11. C. Chen, J. Xi, E. Zhou, L. Peng, Z. Chen et al., Porous graphene microflowers for high-performance microwave absorption. Nano-Micro Lett. 10(2), 26 (2018). https://doi.org/10. 1007/s40820-017-0179-8

12. H. Zhao, Y. Cheng, H. Lv, G. Ji, Y. Du, A novel hierarchically porous magnetic carbon derived from biomass for strong lightweight microwave absorption. Carbon 142, 245-253 (2019). https://doi.org/10.1016/j.carbon.2018.10.027

13. P. Liu, C. Zhu, S. Gao, C. Guan, Y. Huang et al., N-doped porous carbon nanoplates embedded with $\mathrm{CoS} 2$ vertically anchored on carbon cloths for flexible and ultrahigh microwave absorption. Carbon 163, 348-359 (2020). https://doi.org/ 10.1016/j.carbon.2020.03.041

14. D. Ding, Y. Wang, X. Li, R. Qiang, P. Xu et al., Rational design of core-shell Co@ C microspheres for high-performance microwave absorption. Carbon 111, 722-732 (2017). https://doi.org/10.1016/j.carbon.2016.10.059

15. Q. Liu, Q.Cao, H. Bi, C. Liang, K. Yuan et al., CoNi@ $\mathrm{SiO}_{2} @$ $\mathrm{TiO}_{2}$ and $\mathrm{CoNi} @$ Air@ $\mathrm{TiO}_{2}$ microspheres with strong wideband microwave absorption. Adv. Mater. 28(3), 486-490 (2016). https://doi.org/10.1002/adma.201503149

16. X. Wang, T. Zhu, S. Chang, Y. Lu, W. Mi et al., 3D nest-like architecture of core-shell $\mathrm{CoFe}_{2} \mathrm{O}_{4} @ 1 \mathrm{~T} / 2 \mathrm{H}-\mathrm{MoS}_{2}$ composites with tunable microwave absorption performance. ACS Appl. Mater. Interfaces 12(9), 11252-11264 (2020). https://doi.org/ 10.1021/acsami.9b23489
17. R. Shu, Y. Wu, W. Li, J. Zhang, Y. Liu et al., Fabrication of ferroferric oxide-carbon/reduced graphene oxide nanocomposites derived from Fe-based metal-organic frameworks for microwave absorption. Compos. Sci. Technol. 196, 108240 (2020). https://doi.org/10.1016/j.compscitech.2020.108240

18. X. Xu, G. Wang, G. Wan, S. Shi, C. Hao et al., Magnetic Ni/ graphene connected with conductive carbon nano-onions or nanotubes by atomic layer deposition for lightweight and lowfrequency microwave absorption. Chem. Eng. J. 382, 122980 (2020). https://doi.org/10.1016/j.cej.2019.122980

19. Z. Yang, M. Li, L. Yang, J. Liu, Y. Wang et al., Constructing uniform $\mathrm{Fe}_{3} \mathrm{O}_{4} @ \mathrm{C} @ \mathrm{MnO}_{2}$ microspheres with yolk-shell interior toward enhancement in microwave absorption. J. Alloys Compd. 817, 152795 (2020). https://doi.org/10.1016/j.jallcom. 2019.152795

20. X. Liu, C. Hao, L. He, C. Yang, Y. Chen et al., Yolk-shell structured $\mathrm{Co}-\mathrm{C} / \mathrm{Void} / \mathrm{Co}_{9} \mathrm{~S}_{8}$ composites with a tunable cavity for ultrabroadband and efficient low-frequency microwave absorption. Nano Res. 11(8), 4169-4182 (2018). https://doi. org/10.1007/s12274-018-2006-Z

21. P. Liu, S. Gao, X. Liu, Y. Huang, W. He et al., Rational construction of hierarchical hollow CuS@ $\mathrm{CoS}_{2}$ nanoboxes with heterogeneous interfaces for high-efficiency microwave absorption materials. Compos. Pt. B-Eng. 192, 107992 (2020). https://doi.org/10.1016/j.compositesb.2020.107992

22. Z. Wu, K. Pei, L. Xing, X. Yu, W. You et al., Enhanced microwave absorption performance from magnetic coupling of magnetic nanoparticles suspended within hierarchically tubular composite. Adv. Funct. Mater. 29(28), 1901448 (2019). https://doi.org/10.1002/adfm.201901448

23. T. Hou, B. Wang, M. Ma, A. Feng, Z. Huang et al., Preparation of two-dimensional titanium carbide $\left(\mathrm{Ti}_{3} \mathrm{C}_{2} \mathrm{~T}_{\mathrm{x}}\right)$ and $\mathrm{NiCo}_{2} \mathrm{O}_{4}$ composites to achieve excellent microwave absorption properties. Compos. Pt. B-Eng. 180, 107577 (2020). https://doi.org/ 10.1016/j.compositesb.2019.107577

24. N. Yang, Z.-X. Luo, S.-C. Chen, G. Wu, Y.-Z. Wang, $\mathrm{Fe}_{3} \mathrm{O}_{4}$ nanoparticle/n-doped carbon hierarchically hollow microspheres for broadband and high-performance microwave absorption at an ultralow filler loading. ACS Appl. Mater. Interfaces 12(16), 18952-18963 (2020). https://doi.org/10. 1021/acsami.0c04185

25. Z. Hou, J. Xue, H. Wei, X. Fan, F. Ye et al., Tailorable microwave absorption properties of RGO/SiC/CNT nanocomposites with 3D hierarchical structure. Ceram. Int. 46(11), 1816018167 (2020). https://doi.org/10.1016/j.ceramint.2020.04.137

26. L. Liu, N. He, T. Wu, P. Hu, G. Tong, $\mathrm{Co} / \mathrm{C} / \mathrm{Fe} / \mathrm{C}$ hierarchical flowers with strawberry-like surface as surface plasmon for enhanced permittivity, permeability, and microwave absorption properties. Chem. Eng. J. 355, 103-108 (2019). https:// doi.org/10.1016/j.cej.2018.08.131

27. Y. Zhang, H. Meng, Y. Shi, X. Zhang, C. Liu et al., TiN/Ni/C ternary composites with expanded heterogeneous interfaces for efficient microwave absorption. Compos. Pt. B-Eng. 193, 108028 (2020). https://doi.org/10.1016/j.compositesb.2020. 108028 
28. L. Yan, M. Zhang, S. Zhao, T. Sun, B. Zhang et al., Wire-intube ZnO@ carbon by molecular layer deposition: accurately tunable electromagnetic parameters and remarkable microwave absorption. Chem. Eng. J. 382, 122860 (2020). https:// doi.org/10.1016/j.cej.2019.122860

29. L. Xing, X. Li, Z. Wu, X. Yu, J. Liu et al., 3D hierarchical local heterojunction of $\mathrm{MoS}_{2} / \mathrm{FeS}_{2}$ for enhanced microwave absorption. Chem. Eng. J. 379, 122241 (2020). https://doi.org/ 10.1016/j.cej.2019.122241

30. J. Wang, L. Liu, S. Jiao, K. Ma, J. Lv et al., Hierarchical carbon fiber@MXene@ $\mathrm{MoS}_{2}$ core-sheath synergistic microstructure for tunable and efficient microwave absorption. Adv. Funct. Mater. 30(45), 2002595 (2020). https://doi.org/10.1002/ adfm.202002595

31. X. Liang, Z. Man, B. Quan, J. Zheng, W. Gu et al., Environment-stable $\mathrm{Co}_{\mathrm{x}} \mathrm{Ni}_{\mathrm{y}}$ encapsulation in stacked porous carbon nanosheets for enhanced microwave absorption. NanoMicro Lett. 12(1), 102 (2020). https://doi.org/10.1007/ s40820-020-00432-2

32. N. Li, G.-W. Huang, Y.-Q. Li, H.-M. Xiao, Q.-P. Feng et al., Enhanced microwave absorption performance of coated carbon nanotubes by optimizing the $\mathrm{Fe}_{3} \mathrm{O}_{4}$ nanocoating structure. ACS Appl. Mater. Interfaces 9(3), 2973-2983 (2017). https:// doi.org/10.1021/acsami.6b13142

33. S. Zhao, Z. Gao, C. Chen, G. Wang, B. Zhang et al., Alternate nonmagnetic and magnetic multilayer nanofilms deposited on carbon nanocoils by atomic layer deposition to tune microwave absorption property. Carbon 98, 196-203 (2016). https://doi. org/10.1016/j.carbon.2015.10.101

34. M. Zhang, Z. Jiang, X. Lv, X. Zhang, Y. Zhang et al., Microwave absorption performance of reduced graphene oxide with negative imaginary permeability. J. Phys. D-Appl. Phys. 53(2), 02LT01 (2019). https://doi.org/10.1088/1361-6463/ab48a7

35. C. Zhou, S. Geng, X. Xu, T. Wang, L. Zhang et al., Lightweight hollow carbon nanospheres with tunable sizes towards enhancement in microwave absorption. Carbon 108, 234-241 (2016). https://doi.org/10.1016/j.carbon.2016.07.015

36. R.C. Che, L.M. Peng, X.F. Duan, Q. Chen, X.L. Liang, Microwave absorption enhancement and complex permittivity and permeability of $\mathrm{Fe}$ encapsulated within carbon nanotubes. Adv. Mater. 16(5), 401-405 (2004). https://doi.org/10.1002/ adma.200306460

37. X. Xu, S. Shi, Y. Tang, G. Wang, M. Zhou et al., Growth of NiAl-layered double hydroxide on graphene toward excellent anticorrosive microwave absorption application. Adv. Sci. 8(5), 2002658 (2021). https://doi.org/10.1002/advs.202002658

38. C. Deng, Y. Sun, L. Pan, T. Wang, Y. Xie et al., Thermal diffusivity of a single carbon nanocoil: uncovering the correlation with temperature and domain size. ACS Nano 10(10), 9710-9719 (2016). https://doi.org/10.1021/acsnano.6b05715

39. H. Ma, K. Nakata, L. Pan, K. Hirahara, Y. Nakayama, Relationship between the structure of carbon nanocoils and their electrical property. Carbon 73, 71-77 (2014). https://doi.org/ 10.1016/j.carbon.2014.02.038

40. Y. Sun, C. Wang, L. Pan, X. Fu, P. Yin et al., Electrical conductivity of single polycrystalline-amorphous carbon nanocoils. Carbon 98, 285-290 (2016). https://doi.org/10. 1016/j.carbon.2015.11.025

41. S. Xu, Z. Fan, S. Yang, Y. Zhao, L. Pan, Flexible, self-powered and multi-functional strain sensors comprising a hybrid of carbon nanocoils and conducting polymers. Chem. Eng. J. 404, 126064 (2021). https://doi.org/10.1016/j.cej.2020.126064

42. C. Deng, L. Pan, D. Zhang, C. Li, H. Nasir, A super stretchable and sensitive strain sensor based on a carbon nanocoil network fabricated by a simple peeling-off approach. Nanoscale 9(42), 16404-16411 (2017). https://doi.org/10. 1039/C7NR05486F

43. R. Cui, L. Pan, D. Zhang, H. Nasir, Electromagnetic microwave absorption properties of carbon nanocoils/tissue. Diam. Relat. Mat. 77, 53-56 (2017). https://doi.org/10.1016/j.diamo nd.2017.05.014

44. Y. Zhao, H. Zhang, X. Yang, H. Huang, G. Zhao et al., In situ construction of hierarchical core-shell $\mathrm{Fe}_{3} \mathrm{O}_{4} @ \mathrm{C}$ nanoparticles-helical carbon nanocoil hybrid composites for highly efficient electromagnetic wave absorption. Carbon 171, 395-408 (2021). https://doi.org/10.1016/j.carbon.2020.09.036

45. G. Wang, Z. Gao, S. Tang, C. Chen, F. Duan et al., Microwave absorption properties of carbon nanocoils coated with highly controlled magnetic materials by atomic layer deposition. ACS Nano 6(12), 11009-11017 (2012). https://doi.org/10.1021/ nn304630h

46. Y. Zhao, J. Wang, H. Huang, T. Cong, S. Yang et al., Growth of carbon nanocoils by porous $\alpha-\mathrm{Fe}_{2} \mathrm{O}_{3} / \mathrm{SnO}_{2}$ catalyst and its buckypaper for high efficient adsorption. Nano-Micro Lett. 12(1), 23 (2020). https://doi.org/10.1007/s40820-019-0365-y

47. Y. Zhao, J. Wang, H. Huang, H. Zhang, T. Cong et al., Catalytic anisotropy induced by multi-particles for growth of carbon nanocoils. Carbon 166, 101-112 (2020). https://doi.org/ 10.1016/j.carbon.2020.05.007

48. H. Huang, L. Xia, Y. Zhao, H. Zhang, T. Cong et al., Threedimensional porous reduced graphene oxide/PEDOT: PSS aerogel: facile preparation and high performance for supercapacitor electrodes. Electrochim. Acta 364, 137297 (2020). https://doi.org/10.1016/j.electacta.2020.137297

49. H. Xu, X. Yin, M. Zhu, M. Li, H. Zhang et al., Constructing hollow graphene nano-spheres confined in porous amorphous carbon particles for achieving full $\mathrm{X}$ band microwave absorption. Carbon 142, 346-353 (2019). https://doi.org/10.1016/j. carbon.2018.10.056

50. R. Shu, J. Zhang, C. Guo, Y. Wu, Z. Wan et al., Facile synthesis of nitrogen-doped reduced graphene oxide/nickel-zinc ferrite composites as high-performance microwave absorbers in the X-band. Chem. Eng. J. 384, 123266 (2020). https://doi. org/10.1016/j.cej.2019.123266

51. J. Zhang, R. Shu, Y. Wu, Z. Wan, M. Zheng, Facile fabrication and enhanced microwave absorption properties of reduced graphene oxide/tin dioxide binary nanocomposites in the X-band. Synth. Met. 257, 116157 (2019). https://doi.org/10.1016/j. synthmet.2019.116157

52. Y. Qin, Y. Zhang, N. Qi, Q. Wang, X. Zhang et al., Preparation of graphene aerogel with high mechanical stability and microwave absorption ability via combining surface support of 
metallic-CNTs and interfacial cross-linking by magnetic nanoparticles. ACS Appl. Mater. Interfaces 11(10), 10409-10417 (2019). https://doi.org/10.1021/acsami.8b22382

53. Y. Zhou, N. Wang, J. Muhammad, D. Wang, Y. Duan et al., Graphene nanoflakes with optimized nitrogen doping fabricated by arc discharge as highly efficient absorbers toward microwave absorption. Carbon 148, 204-213 (2019). https:// doi.org/10.1016/j.carbon.2019.03.034

54. X. Wang, Y. Lu, T. Zhu, S. Chang, W. Wang, $\mathrm{CoFe}_{2} \mathrm{O}_{4} / \mathrm{N}$-doped reduced graphene oxide aerogels for high-performance microwave absorption. Chem. Eng. J. 388, 124317 (2020). https://doi.org/10.1016/j.cej.2020.124317

55. Y. Cheng, P. Hu, S. Zhou, L. Yan, B. Sun et al., Achieving tunability of effective electromagnetic wave absorption between the whole X-band and Ku-band via adjusting PPy loading in $\mathrm{SiC}$ nanowires/graphene hybrid foam. Carbon 132, 430-443 (2018). https://doi.org/10.1016/j.carbon.2018.02.084

56. R. Shu, W. Li, Y. Wu, J. Zhang, G. Zhang, Nitrogen-doped Co-C/MWCNTs nanocomposites derived from bimetallic metal-organic frameworks for electromagnetic wave absorption in the X-band. Chem. Eng. J. 362, 513-524 (2019). https://doi.org/10.1016/j.cej.2019.01.090

57. P. Liu, S. Gao, C. Chen, F. Zhou, Z. Meng et al., Vacanciesengineered and heteroatoms-regulated $\mathrm{N}$-doped porous carbon aerogel for ultrahigh microwave absorption. Carbon 169, 276-287 (2020). https://doi.org/10.1016/j.carbon.2020.07.063

58. S. Dong, X. Zhang, X. Li, J. Chen, P. Hu et al., SiC whiskers-reduced graphene oxide composites decorated with $\mathrm{MnO}$ nanoparticles for tunable microwave absorption. Chem. Eng. J. 392, 123817 (2020). https://doi.org/10.1016/j.cej.2019.123817

59. J. Dong, Y. Lin, H. Zong, H. Yang, L. Wang et al., Threedimensional architecture reduced graphene oxide- $-\mathrm{LiFePO}_{4}$ composite: preparation and excellent microwave absorption performance. Inorg. Chem. 58(3), 2031-2041 (2019). https:// doi.org/10.1021/acs.inorgchem.8b03043

60. J. Xu, X. Zhang, H. Yuan, S. Zhang, C. Zhu et al., N-doped reduced graphene oxide aerogels containing pod-like $\mathrm{N}$-doped carbon nanotubes and FeNi nanoparticles for electromagnetic wave absorption. Carbon 159, 357-365 (2020). https://doi.org/ 10.1016/j.carbon.2019.12.020

61. H. Ji, J. Li, J. Zhang, Y. Yan, Remarkable microwave absorption performance of ultralight graphene-polyethylene glycol composite aerogels with a very low loading ratio of graphene. Compos. Pt. A-Appl. Sci. Manuf. 123, 158-169 (2019). https://doi.org/10.1016/j.compositesa.2019.05.012

62. N. Yang, Z.-X. Luo, G.-R. Zhu, S.-C. Chen, X.-L. Wang et al., Ultralight three-dimensional hierarchical cobalt nanocrystals/N-doped CNTs/carbon sponge composites with a hollow skeleton toward superior microwave absorption. ACS Appl. Mater. Interfaces 11(39), 35987-35998 (2019). https://doi.org/10.1021/acsami.9b11101

63. Y. Wang, X. Gao, Y. Fu, X. Wu, Q. Wang et al., Enhanced microwave absorption performances of polyaniline/graphene aerogel by covalent bonding. Compos. Pt. B-Eng. 169, 221228 (2019). https://doi.org/10.1016/j.compositesb.2019.04.008
64. D. Liu, Y. Du, F. Wang, Y. Wang, L. Cui et al., MOFsderived multi-chamber carbon microspheres with enhanced microwave absorption. Carbon 157, 478-485 (2020). https:// doi.org/10.1016/j.carbon.2019.10.056

65. X. Meng, Y. Liu, G. Han, W. Yang, Y. Yu, Three-dimensional $\left(\mathrm{Fe}_{3} \mathrm{O}_{4} / \mathrm{ZnO}\right) @ \mathrm{C}$ Double-core@ shell porous nanocomposites with enhanced broadband microwave absorption. Carbon 162, 356-364 (2020). https://doi.org/10.1016/j. carbon.2020.02.035

66. J. Tang, N. Liang, L. Wang, J. Li, G. Tian et al., Three-dimensional nitrogen-doped reduced graphene oxide aerogel decorated with Ni nanoparticles with tunable and unique microwave absorption. Carbon 152, 575-586 (2019). https://doi.org/ 10.1016/j.carbon.2019.06.049

67. D. Zhang, Y. Xiong, J. Cheng, J. Chai, T. Liu et al., Synergetic dielectric loss and magnetic loss towards superior microwave absorption through hybridization of few-layer $\mathrm{WS}_{2}$ nanosheets with NiO nanoparticles. Sci. Bull. 65(2), 138-146 (2020). https://doi.org/10.1016/j.scib.2019.10.011

68. Z. Jia, B. Wang, A. Feng, J. Liu, M. Zhang et al., Development of spindle-cone shaped of $\mathrm{Fe} / \alpha-\mathrm{Fe}_{2} \mathrm{O}_{3}$ hybrids and their superior wideband electromagnetic absorption performance. J. Alloys Compd. 799, 216-223 (2019). https://doi.org/10. 1016/j.jallcom.2019.05.336

69. P. Liu, V.M.H. Ng, Z. Yao, J. Zhou, Y. Lei et al., Facile synthesis and hierarchical assembly of flowerlike NiO structures with enhanced dielectric and microwave absorption properties. ACS Appl. Mater. Interfaces 9(19), 16404-16416 (2017). https://doi.org/10.1021/acsami.7b02597

70. L. Wang, M. Liu, G. Wang, B. Dai, F. Yu et al., An ultralight nitrogen-doped carbon aerogel anchored by $\mathrm{Ni}-\mathrm{NiO}$ nanoparticles for enhanced microwave adsorption performance. J. Alloys Compd. 776, 43-51 (2019). https://doi.org/10.1016/j. jallcom.2018.10.214

71. M. Ma, W. Li, Z. Tong, Y. Yang, Y. Ma et al., 1D flower-like $\mathrm{Fe}_{3} \mathrm{O}_{4} @ \mathrm{SiO}_{2} @ \mathrm{MnO}_{2}$ nanochains inducing RGO self-assembly into aerogels for high-efficient microwave absorption. Mater. Des. 188, 108462 (2020). https://doi.org/10.1016/j. matdes.2019.108462

72. Z. Liu, F. Pan, B. Deng, Z. Xiang, W. Lu, Self-assembled $\mathrm{MoS}_{2} / 3 \mathrm{D}$ worm-like expanded graphite hybrids for highefficiency microwave absorption. Carbon 174, 59-69 (2021). https://doi.org/10.1016/j.carbon.2020.12.019

73. Z. Wang, R. Wei, J. Gu, H. Liu, C. Liu et al., Ultralight, highly compressible and fire-retardant graphene aerogel with selfadjustable electromagnetic wave absorption. Carbon 139, 11261135 (2018). https://doi.org/10.1016/j.carbon.2018.08.014

74. Y. Jiang, Y. Chen, Y.-J. Liu, G.-X. Sui, Lightweight spongy bone-like graphene@SiC aerogel composites for high-performance microwave absorption. Chem. Eng. J. 337, 522-531 (2018). https://doi.org/10.1016/j.cej.2017.12.131

75. Y. Li, X. Liu, X. Nie, W. Yang, Y. Wang et al., Multifunctional organic-inorganic hybrid aerogel for self-cleaning, heat-insulating, and highly efficient microwave absorbing material. Adv. Funct. Mater. 29(10), 1807624 (2019). https://doi.org/10.1002/ adfm.201807624 
76. F. Ye, Q. Song, Z. Zhang, W. Li, S. Zhang et al., Direct growth of edge-rich graphene with tunable dielectric properties in porous $\mathrm{Si}_{3} \mathrm{~N}_{4}$ ceramic for broadband high-performance microwave absorption. Adv. Funct. Mater. 28(17), 1707205 (2018). https://doi.org/10.1002/adfm.201707205

77. L. Wang, M. Huang, X. Yu, W. You, J. Zhang et al., MOFderived $\mathrm{Ni}_{1-\mathrm{x}} \mathrm{Co}_{\mathrm{x}} @$ carbon with tunable nano-microstructure as lightweight and highly efficient electromagnetic wave absorber. Nano-Micro Lett. 12(1), 150 (2020). https://doi.org/ 10.1007/s40820-020-00488-0

78. L. Wang, B. Wen, H. Yang, Y. Qiu, N. He, Hierarchical nestlike structure of $\mathrm{Co} / \mathrm{Fe} \mathrm{MOF}$ derived $\mathrm{CoFe} @ \mathrm{C}$ composite as wide-bandwidth microwave absorber. Compos. Part A-Appl. Sci. Manuf. 135, 105958 (2020). https://doi.org/10.1016/j. compositesa.2020.105958

79. H.-B. Zhao, J.-B. Cheng, J.-Y. Zhu, Y.-Z. Wang, Ultralight $\mathrm{CoNi} / \mathrm{rGO}$ aerogels toward excellent microwave absorption at ultrathin thickness. J. Mater. Chem. C 7(2), 441-448 (2019). https://doi.org/10.1039/C8TC05239E

80. M. Zhang, H. Ling, S. Ding, Y. Xie, T. Cheng et al., Synthesis of CF@PANI hybrid nanocomposites decorated with $\mathrm{Fe}_{3} \mathrm{O}_{4}$ nanoparticles towards excellent lightweight microwave absorber. Carbon 174, 248-259 (2021). https://doi.org/10. 1016/j.carbon.2020.12.005

81. Z. Li, H. Lin, S. Ding, H. Ling, T. Wang et al., Synthesis and enhanced electromagnetic wave absorption performances of $\mathrm{Fe}_{3} \mathrm{O}_{4} @ \mathrm{C}$ decorated walnut shell-derived porous carbon.
Carbon 167, 148-159 (2020). https://doi.org/10.1016/j.carbon. 2020.05.070

82. H. Zhang, Y. Zhao, X. Yang, G. Zhao, D. Zhang et al., A facile synthesis of novel amorphous $\mathrm{TiO}_{2}$ nanorods decorated rGO hybrid composites with wide band microwave absorption. Nanomaterials 10(11), 2141 (2020). https://doi.org/10.3390/ nano10112141

83. Y. Zhao, H. Zhang, T. Cong, H. Huang, J. Muhammad et al., Crystallization-and morphology-tunable $\mathrm{Fe}_{3} \mathrm{O}_{4} @ \mathrm{C}$ core-shell composites decorated on carbon nanotube skeleton with tailorable electromagnetic wave absorption behavior. Appl. Phys. Express 13(12), 125501 (2020). https://doi.org/10.35848/ 1882-0786/abc491

84. P. Liu, S. Gao, Y. Wang, F. Zhou, Y. Huang et al., Core-shell

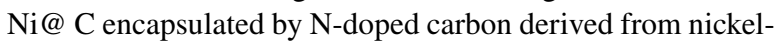
organic polymer coordination composites with enhanced microwave absorption. Carbon 170, 503-516 (2020). https:// doi.org/10.1016/j.carbon.2020.08.043

85. P. Liu, S. Gao, G. Zhang, Y. Huang, W. You et al., Hollow engineering to $\mathrm{Co@N-doped} \mathrm{carbon} \mathrm{nanocages} \mathrm{via} \mathrm{synergistic} \mathrm{pro-}$ tecting-etching strategy for ultrahigh microwave absorption. Adv. Funct. Mater. (2021). https://doi.org/10.1002/adfm.202102812

86. S. Gao, G. Zhang, Y. Wang, X. Han, Y. Huang et al., MOFs derived magnetic porous carbon microspheres constructed by core-shell Ni@C with high-performance microwave absorption. J. Mater. Sci. Technol. 88, 56-65 (2021). https://doi.org/ 10.1016/j.jmst.2021.02.011 